YITP-14-22

\title{
Cosmological Scaling Solutions for Multiple Scalar Fields
}

\author{
Takeshi Chiba, ${ }^{1}$ Antonio De Felice, ${ }^{2}$ and Shinji Tsujikawa ${ }^{3}$ \\ ${ }^{1}$ Department of Physics, College of Humanities and Sciences, Nihon University, Tokyo 156-8550, Japan \\ ${ }^{2}$ Yukawa Institute for Theoretical Physics, Kyoto University, Kyoto 606-8502, Japan \\ ${ }^{3}$ Department of Physics, Faculty of Science, Tokyo University of Science, \\ 1-3, Kagurazaka, Shinjuku-ku, Tokyo 162-8601, Japan
}

(Dated: May 29, 2022)

\begin{abstract}
The general k-essence Lagrangian for the existence of cosmological scaling solutions is derived in the presence of multiple scalar fields coupled to a barotropic perfect fluid. In addition to the scaling fixed point associated with the dynamics during the radiation and matter eras, we also obtain a scalar-field dominated solution relevant to dark energy and discuss the stability of them in the twofield set-up. We apply our general results to a model of two canonical fields with coupled exponential potentials arising in string theory. Depending on model parameters and initial conditions, we show that the scaling matter-dominated epochs followed by an attractor with cosmic acceleration can be realized with/without the couplings to scalar fields. The different types of scaling solutions can be distinguished from each other by the evolution of the dark energy equation of state from high-redshifts to today.
\end{abstract}

\section{INTRODUCTION}

The scalar fields may have played important roles for the expansion history of the Universe. The slow-rolling scalar field along a nearly flat potential drives the accelerated expansion in the early Universe-dubbed inflation [1]. The theoretical prediction of inflation for the generation of density perturbations from the quantum fluctuation of a scalar degree of freedom is consistent with the Cosmic Microwave Background (CMB) temperature anisotropies observed by the Planck satellite [2]. The scalar fields can be also responsible for dark energy at the expense of having a very light mass of the order of the today's Hubble constant $H_{0} \simeq 10^{-33} \mathrm{eV}[3,4]$.

The energy scales of scalar fields appearing in particle physics are usually much higher than the present cosmological density [5]. The dominance of the field energy density $\rho_{x}$ over the background energy density $\rho_{m}$ in the early Universe after inflation contradicts with the successful cosmological sequence of the radiation, matter, and accelerated epochs. If there is a scaling solution where $\rho_{x}$ is proportional to $\rho_{m}$, however, the Universe can enter the regime in which the field energy density is sub-dominant to the total energy density.

It is well known that a canonical field with the exponential potential $V(\phi)=V_{0} e^{-\lambda \phi}$ gives rise to scaling solutions with $\rho_{x} / \rho_{m}=$ constant [6] 8 ] (where the reduced Planck mass $M_{\mathrm{pl}}$ is set to unity). Provided that the constant $\lambda$ satisfies the condition $\lambda^{2}>3\left(1+w_{m}\right)$, where $w_{m}$ is the equation of state of the background barotropic fluid, the solutions approach the scaling attractor characterized by the field density parameter $\Omega_{x}=3\left(1+w_{m}\right) / \lambda^{2}$ [7]. In the radiation-dominated epoch there is the bound $\Omega_{x}<0.045$ from the Big-Bang-Nucleosynthesis (BBN) [9], so the primordial scaling field is compatible with the data for $\lambda>9.4$.

The scaling solutions also arise for non-canonical scalar-field models with the Lagrangian $P(\phi, X)$ [10, 11], where $X$ is a kinetic term of the field $\phi$. For the existence of scaling solutions the Lagrangian is constrained to be in the form $P=X g(Y)$, where $g$ is an arbitrary function in terms of $Y \equiv X e^{\lambda \phi}$ and $\lambda$ is a constant 12, 13] (see also Refs. [14, 15]). This accommodates the case in which the coupling $Q$ between the field $\phi$ and the barotropic fluid is present. In the absence of the coupling, the field density parameter relevant to scaling solutions during the radiation or matter eras is given by $\Omega_{x}=3\left(1+w_{m}\right) P_{, X} / \lambda^{2}$, where $P_{, X}=\partial P / \partial X$ [16, 17].

For the Lagrangian $P=X g(Y)$, there exists a scalar-field dominated solution $\left(\Omega_{x}=1\right)$ characterized by the effective equation of state $w_{\text {eff }}=-1+\lambda^{2} /\left(3 P_{, X}\right)$ [16]. With this solution the accelerated expansion can be realized for $\lambda^{2} / P_{, X}<2$. In this case, however, we do not have a physically meaningful scaling solution with $\Omega_{x}<1$ during the radiation and matter eras. There are several possible ways of realizing a transition from the scaling regime to the epoch of cosmic acceleration. One is to introduce a canonical single field composed by the sum of exponential potentials, e.g., $V(\phi)=V_{1} e^{-\lambda_{1} \phi}+V_{2} e^{-\lambda_{2} \phi}$ satisfying $\lambda_{1}^{2}>3\left(1+w_{m}\right)$ and $\lambda_{2}^{2}<2$ [18] (see also Refs. [19]). The joint analysis based on the data of supernovae type Ia, CMB, and baryonic acoustic oscillations showed that the two slopes are constrained to be $\lambda_{1}>11.7$ and $\lambda_{2}<0.539$ (95\% CL) [20].

Another way out is to introduce multiple canonical scalar fields $\phi_{i}(i=1,2, \cdots, N)$ with the sum of exponential potentials, i.e., $V=\sum_{i=1}^{N} V_{i} e^{-\lambda_{i} \phi_{i}}[21$, 22]. In this case there exists a so-called assisted inflationary solution characterized by the effective equation of state $w_{\text {eff }}=-1+\lambda^{2} / 3$, where $\lambda \equiv\left(\sum_{i=1}^{N} 1 / \lambda_{i}^{2}\right)^{-1 / 2}$. Even if each field is unable to be responsible for cosmic acceleration (i.e., $\lambda_{i}^{2}>2$ ), multiple scalar fields can cooperate to give dynamics matching a 
single-field solution with $\lambda^{2}<2$. In this model, the dynamics of scaling solutions followed by the assisted dark energy attractor has been studied in Refs. [23 27].

For the system of multiple fields with the more general Lagrangian $P=\sum_{i=1}^{N} X_{i} g\left(Y_{i}\right)$, where $g$ is an arbitrary function with respect to $Y_{i} \equiv X_{i} e^{\lambda_{i} \phi_{i}}$, it was shown in Ref. 26] that the scaling radiation/matter eras can be followed by the assisted inflationary attractor. However, this analysis does not cover the models in which the multiple scalar fields are coupled each other 28 30].

Recently, Dodelson et al. [30] presented a string-theoretic model described by canonical fields with the coupled potential $V=\sum_{i=1}^{2} V_{i} e^{\alpha_{i} \phi_{1}+\beta_{i} \phi_{2}}$. This multi-field theory follows from a torus compactification of an overall volume in the presence of a dilaton field. Dodelson et al. showed the existence of not only scaling solutions but also solutions with the accelerated expansion relevant to dark energy. In general there are many scalar fields present in string theory (dilaton, moduli, axion), so the action in the Einstein frame contains coupled scalar fields after a suitable compactification. It is also known that $\alpha^{\prime}$ corrections to the tree-level string action give rise to higher-order derivative terms such as $X^{2}$ [10]. Then, the general Einstein-frame action with $N$ scalar fields can be described by $P\left(\phi_{1}, \cdots, \phi_{N}, X_{1}, \cdots, X_{N}\right)$.

In this paper, we study the construction of the multi-field Lagrangian $P\left(\phi_{1}, \cdots, \phi_{N}, X_{1}, \cdots, X_{N}\right)$ that possesses scaling solutions. For generality we also introduce the couplings between the scalar fields and the background barotropic fluid. The resulting Lagrangian for the existence of scaling solutions is surprisingly simple and it covers the multi-field models studied in Refs. 28 30] as specific cases. Moreover, the presence of multiple scalar fields allows the transition from the scaling radiation/matter eras to the epoch of cosmic acceleration.

For the general multi-field scaling Lagrangian we derive the autonomous equations of motion and the fixed points relevant to dark energy as well as the scaling solution. We show the existence of scalar-field dominated solutions $\left(\Omega_{x}=1\right)$ having the property of assisted inflation. For the scaling solution relevant to the dynamics during the radiation and matter eras, we also obtain analytic expressions for the field density parameter $\Omega_{x}$ and the field equation of state $w_{x}$. The stability of such fixed points will be discussed by considering linear perturbations about them.

As an application of our general scaling Lagrangian, we also study the cosmology for a two-field model described by canonical fields with the potential $V=\sum_{i=1}^{2} V_{i} e^{\alpha_{i} \phi_{1}+\beta_{i} \phi_{2}}$ in the absence/presence of couplings between the fields and the background barotropic fluid. In such a model there exist scaling and accelerated fixed points other than those discussed above. We show how the scaling radiation or matter eras (including the $\phi$-matter-dominated-epoch [31]) can be followed by the scalar-field dominated attractor with cosmic acceleration.

This paper is organized as follows. In Sec. I we derive the multi-field Lagrangian that possesses scaling solutions characterized by $\rho_{x} / \rho_{m}=$ constant. In Sec. II we obtain autonomous equations and some physically important fixed points of the general multi-field scaling Lagrangian. In Sec. IV the stability of fixed points for the scaling solution and the accelerated scalar-field dominated point will be discussed. In Sec. $\nabla$ we study the cosmological dynamics for two canonical fields with the potential $V=\sum_{i=1}^{2} V_{i} e^{\alpha_{i} \phi_{1}+\beta_{i} \phi_{2}}$ in detail. Sec. VI is devoted to conclusions.

\section{GENERAL MULTI-FIELD LAGRANGIAN FOR THE EXISTENCE OF SCALING SOLUTIONS}

We start with the k-essence model [10, 11] described by $N$ scalar fields $\phi_{i}(i=1,2, \cdots, N)$ with kinetic energies $X_{i} \equiv-(1 / 2) g^{\mu \nu} \partial_{\mu} \phi_{i} \partial_{\nu} \phi_{i}$, where $g^{\mu \nu}$ is the metric tensor. We also take into account the matter Lagrangian $\mathcal{L}_{m}$ coupled to the scalar fields. The action in such a system is given by

$$
S=\int d^{4} x \sqrt{-g}\left[\frac{1}{2} R+P\left(\phi_{1}, \cdots \phi_{N}, X_{1}, \cdots, X_{N}\right)\right]+\int d^{4} x \mathcal{L}_{m}\left(\phi_{1}, \cdots \phi_{N}, g_{\mu \nu}\right),
$$

where $g$ is the determinant of the metric $g_{\mu \nu}, P$ is the Lagrangian of multiple scalar fields, and $\mathcal{L}_{m}$ is the matter Lagrangian. We use the unit $M_{\mathrm{pl}}=1$.

We introduce couplings between the scalar fields and matter in the form

$$
Q_{i}\left(\phi_{i}\right)=-\frac{1}{\rho_{m}} \frac{1}{\sqrt{-g}} \frac{\partial \mathcal{L}_{m}}{\partial \phi_{i}} \quad(i=1,2, \cdots, N),
$$

where $\rho_{m}$ is the energy density of matter. The couplings $Q_{i}$ are analogous to those appearing in the coupled quintessence scenario discussed in Refs. [12, 31]. In Brans-Dicke (BD) theory [32], for example, the BD scalar field couples to non-relativistic matter with constant couplings $Q_{i}$ in the Einstein frame [33 36]. In such theory the radiation does not couple to the BD scalar because it is traceless. In the following we consider a barotropic perfect fluid with the constant equation of state $w_{m}$ coupled to the fields $\phi_{i}$. Although we basically have the matter-dominated epoch in mind, Our analysis also covers the radiation era by setting $Q_{i}$ zero for radiation. In the rest of the paper we assume that $w_{m}$ is in the range $0 \leq w_{m}<1$. 


\section{A. Background equations of motion}

Let us study the cosmology on the flat Friedmann-Lemaître-Robertson-Walker background with the line-element $d s^{2}=-d t^{2}+a^{2}(t) \delta_{i j} d x^{i} d x^{j}$, where $a(t)$ is the scale factor with cosmic time $t$. Varying the action (2.1) with respect to $\phi_{i}$, we obtain the following equations of motion for $N$ scalar fields

$$
\frac{d}{d t}\left(\dot{\phi}_{i} P_{, X_{i}}\right)+3 H \dot{\phi}_{i} P_{X_{i}}-P_{,_{i}}=-Q_{i} \rho_{m}, \quad(i=1,2, \cdots, N),
$$

where a dot represents a derivative with respect to $t, H \equiv \dot{a} / a, P_{,_{X}} \equiv \partial P / \partial X_{i}$, and $P_{,_{i}} \equiv \partial P / \partial \phi_{i}$. In the following we assume that the couplings $Q_{i}$ are constant ${ }^{1}$.

Multiplying the term $\dot{\phi}_{i}$ for Eq. (2.3) and summing up each equation from $i=1$ to $i=N$, it follows that

$$
\dot{\rho}+3 H\left(1+w_{x}\right) \rho=-\sum_{i=1}^{N} Q_{i} \rho_{m} \dot{\phi}_{i}
$$

where

$$
\rho \equiv \sum_{i=1}^{N} 2 X_{i} P_{, X_{i}}-P, \quad w_{x} \equiv P / \rho
$$

Due to the energy conservation the barotropic perfect fluid obeys

$$
\dot{\rho}_{m}+3 H\left(1+w_{m}\right) \rho_{m}=\sum_{i=1}^{N} Q_{i} \rho_{m} \dot{\phi}_{i}
$$

From the Einstein equations we obtain

$$
\begin{aligned}
& 3 H^{2}=\rho+\rho_{m} \\
& 2 \dot{H}=-\left(1+w_{x}\right) \rho-\left(1+w_{m}\right) \rho_{m} .
\end{aligned}
$$

We also define the effective equation of state

$$
w_{\mathrm{eff}} \equiv-1-\frac{2 \dot{H}}{3 H^{2}} .
$$

From Eqs. (2.7) and (2.8) we can express $w_{\text {eff }}$ in the form

$$
w_{\text {eff }}=w_{m}+\left(w_{x}-w_{m}\right) \Omega_{x}, \quad \text { where } \quad \Omega_{x} \equiv \frac{\rho}{\rho+\rho_{m}} .
$$

\section{B. Derivation of the multi-field scaling Lagrangian}

Let us derive the Lagrangian that gives rise to scaling solutions characterized by $\rho / \rho_{m}=C$, where $C$ is a non-zero constant. This demands the condition

$$
\frac{\rho^{\prime}}{\rho}=\frac{\rho_{m}^{\prime}}{\rho_{m}},
$$

where a prime represents a derivative with respect to the e-folding number $\mathcal{N}=\ln a($ having the relation $d \mathcal{N} / d t=H)$. On using the relations (2.4) and (2.6), it follows that

$$
\sum_{i=1}^{N} Q_{i} \phi_{i}^{\prime}=3\left(w_{m}-w_{x}\right) \Omega_{x},
$$

\footnotetext{
${ }^{1}$ Along the line of Ref. [17], it is also possible to generalize the analysis to the case in which the couplings depend on scalar fields.
} 
where

$$
(\ln \rho)^{\prime}=\left(\ln \rho_{m}\right)^{\prime}=-3\left(1+w_{\text {eff }}\right) .
$$

In the scaling regime where $w_{x}$ and $\Omega_{x}$ are constant, we can integrate Eq. (2.13) to give

$$
\rho \propto \rho_{m} \propto a^{-3\left(1+w_{\text {eff }}\right)} .
$$

Since $H^{2} \propto \rho \propto a^{-3\left(1+w_{\text {eff }}\right)}$, the scale factor and the Hubble parameter evolve, respectively, as

$$
a \propto t^{2 /\left[3\left(1+w_{\text {eff }}\right)\right]}, \quad H=\frac{2}{3\left(1+w_{\text {eff }}\right) t} .
$$

Each term on the left-hand side (lhs) of Eq. (2.12) can be written in the form $Q_{i} \phi_{i}^{\prime}=3\left(w_{m}-w_{x}\right) \Omega_{x} r_{i}$, where $\sum_{i=1}^{N} r_{i}=1$. The ratios $r_{i}$ should be constant in the scaling regime. On using Eq. (2.10), it follows that

$$
\phi_{i}^{\prime}=\frac{3\left(w_{m}-w_{\mathrm{eff}}\right) r_{i}}{Q_{i}} .
$$

Then the field kinetic energies have the dependence $X_{i}=H^{2} \phi_{i}^{\prime 2} / 2 \propto H^{2} \propto \rho$, so that

$$
\left(\ln X_{i}\right)^{\prime}=-3\left(1+w_{\text {eff }}\right) .
$$

Moreover, since $w_{x}=P / \rho$ is constant in the scaling regime, the pressure $P$ has the same dependence as $\rho$. Hence we have

$$
(\ln P)^{\prime}=\sum_{i=1}^{N}\left[\frac{\partial \ln P}{\partial \phi_{i}} \phi_{i}^{\prime}+\frac{\partial \ln P}{\partial \ln X_{i}}\left(\ln X_{i}\right)^{\prime}\right]=-3\left(1+w_{\mathrm{eff}}\right) .
$$

Substitution of Eqs. (2.16) and (2.17) into Eq. (2.18) reads

$$
\sum_{i=1}^{N}\left(\frac{\partial \ln P}{\partial \ln X_{i}}-\frac{1}{\lambda_{i}} \frac{\partial \ln P}{\partial \phi_{i}}\right)=1
$$

where

$$
\lambda_{i} \equiv \frac{\left(1+w_{\mathrm{eff}}\right) Q_{i}}{\left(w_{m}-w_{\mathrm{eff}}\right) r_{i}}
$$

Integrating Eq. (2.19), we obtain the Lagrangian

$$
P=e^{-\lambda_{1} \phi_{1}} g\left(Y_{1}, \cdots, Y_{N}, Z_{1}, \cdots, Z_{N-1}\right),
$$

where $g$ is an arbitrary function with respect to

$$
Y_{i} \equiv X_{i} e^{\lambda_{1} \phi_{1}}, \quad Z_{i} \equiv \phi_{i+1}-\frac{\lambda_{1}}{\lambda_{i+1}} \phi_{1} .
$$

In the presence of the field $\phi_{1}$ alone we have $Y_{i}=0(i \geq 2)$ and $Z_{i}=0(i \geq 1)$ (because $r_{i} \rightarrow 0$ and $\lambda_{i} \rightarrow \infty$ for $i \geq 2$ ), in which case the Lagrangian (2.21) reduces to $\bar{P}=e^{-\lambda_{1} \phi_{1}} g\left(X_{1} e^{\lambda_{1} \phi_{1}}\right)$. This corresponds to the single-field scaling Lagrangian ${ }^{2}$ derived in Refs. [12, 13].

In the scaling regime we have $\phi_{i}^{\prime}=3\left(1+w_{\text {eff }}\right) / \lambda_{i}=$ constant from Eqs. (2.16) and (2.20). Integrating this equation and using Eq. (2.15), each field evolves as

$$
\phi_{i}=\phi_{i}^{(0)}+\frac{2}{\lambda_{i}} \ln \left(\frac{t}{t_{0}}\right),
$$

where $\phi_{i}^{(0)}$ is the field value at $t=t_{0}$. Along this solution the quantities $Y_{i}$ and $Z_{i}$ behave as

$$
\begin{aligned}
Y_{i} & =\frac{1}{2} \dot{\phi}_{i}^{2} e^{\lambda_{1} \phi_{1}} \propto t^{-2} t^{2}=\text { constant } \\
Z_{i} & =\phi_{i+1}^{(0)}-\frac{\lambda_{1}}{\lambda_{i+1}} \phi_{1}^{(0)}=\text { constant } .
\end{aligned}
$$

Hence the function $g$ remains constant for the scaling solution discussed above.

\footnotetext{
${ }^{2}$ Refs. [12, 13] derived the Lagrangian in the form $P=X_{1} g\left(X_{1} e^{\lambda_{1} \phi_{1}}\right)$, but this is equivalent to $P=e^{-\lambda_{1} \phi_{1}} g\left(X_{1} e^{\lambda_{1} \phi_{1}}\right)$.
} 


\section{Specific scaling models}

The Lagrangian (2.21) is general enough to cover a wide variety of multi-field models having scaling solutions.

Let us first consider the two-field model described by

$$
g\left(Y_{1}, Y_{2}, Z_{1}\right)=Y_{1}+Y_{2}-V_{1} e^{\mu_{1} Z_{1}}-V_{2} e^{\mu_{2} Z_{1}},
$$

where $V_{i}$ and $\mu_{i}(i=1,2)$ are constants. Then the Lagrangian (2.21) reads

$$
P=X_{1}+X_{2}-V_{1} e^{\alpha_{1} \phi_{1}+\mu_{1} \phi_{2}}-V_{2} e^{\alpha_{2} \phi_{1}+\mu_{2} \phi_{2}},
$$

where

$$
\alpha_{1} \equiv-\lambda_{1}\left(1+\mu_{1} / \lambda_{2}\right) \quad \alpha_{2} \equiv-\lambda_{1}\left(1+\mu_{2} / \lambda_{2}\right) .
$$

This is the model appearing in string theory [30].

Under the scaling transformation with a parameter $\mathcal{A}$ :

$$
\begin{aligned}
& \phi_{1} \rightarrow \phi_{1}-2 \frac{\mu_{1}-\mu_{2}}{\alpha_{2} \mu_{1}-\alpha_{1} \mu_{2}} \mathcal{A}=\phi_{1}+\frac{2 \mathcal{A}}{\lambda_{1}}, \\
& \phi_{2} \rightarrow \phi_{2}+2 \frac{\alpha_{1}-\alpha_{2}}{\alpha_{2} \mu_{1}-\alpha_{1} \mu_{2}} \mathcal{A}=\phi_{2}+\frac{2 \mathcal{A}}{\lambda_{2}},
\end{aligned}
$$

the sum of the two potentials in Eq. (2.27) transforms homogeneously: $V \rightarrow V e^{-2 \mathcal{A}}$ [30]. The scaling solution corresponds to the case in which each term in Eq. (2.27) evolves in the same way as $H^{2} \propto t^{-2}$. On using the transformation (2.30), we find that the scaling solution (2.23) has such a property. We stress that the variables $\lambda_{i}$ $(i=1,2)$ are more fundamental than $\alpha_{i}$ to determine the property of scaling solutions. The variables $\mu_{1}$ and $\mu_{2}$ also matter when we discuss the stability of the solution (2.23).

We can also consider more general models with two canonical fields described by

$$
g\left(Y_{1}, Y_{2}, Z_{1}\right)=Y_{1}+Y_{2}-h\left(Z_{1}\right)
$$

where $h\left(Z_{1}\right)$ is an arbitrary function with respect to $Z_{1}$. In this case the field has a potential of the form

$$
V\left(\phi_{1}, \phi_{2}\right)=e^{-\lambda_{1} \phi_{1}} h\left(\phi_{2}-\frac{\lambda_{1}}{\lambda_{2}} \phi_{1}\right)
$$

It is instructive to define the field $\sigma$ along the scaling solution and the orthogonal field $s$ in terms of their velocities as [37, 38]

$$
\begin{aligned}
\dot{\sigma} & =(\cos \theta) \dot{\phi}_{1}+(\sin \theta) \dot{\phi}_{2}, \\
\dot{s} & =-(\sin \theta) \dot{\phi}_{1}+(\cos \theta) \dot{\phi}_{2} .
\end{aligned}
$$

where $\cos \theta \equiv \dot{\phi}_{1} / \sqrt{\dot{\phi}_{1}^{2}+\dot{\phi}_{2}^{2}}$ and $\sin \theta \equiv \dot{\phi}_{2} / \sqrt{\dot{\phi}_{1}^{2}+\dot{\phi}_{2}^{2}}$. The scaling solution (2.23) obeys the following relations

$$
\cos \theta=\frac{\lambda}{\lambda_{1}}, \quad \sin \theta=\frac{\lambda}{\lambda_{2}},
$$

where $1 / \lambda^{2}=1 / \lambda_{1}^{2}+1 / \lambda_{2}^{2}$. Since $\theta$ is constant, the trajectory in field space is a straight line. The velocity $\dot{s}$ of the orthogonal field vanishes by definition. Then the scaling solution in the two-field system can be described by an effective single-field trajectory with

$$
\dot{\sigma}=\frac{2}{\lambda t}, \quad \sigma=\sigma^{(0)}+\frac{2}{\lambda} \ln \left(\frac{t}{t_{0}}\right)
$$

where $\sigma^{(0)}$ is a constant. The effective slope $\lambda$, which is the combination of $\lambda_{1}$ and $\lambda_{2}$, is crucial to determine the property of scaling solutions. This is also the case for more general non-canonical fields described by the Lagrangian (2.21). We shall study this issue in detail in the next section. 
The above prescription can be generalized to the $N$-scalar field models with

$$
g\left(Y_{1}, \cdots, Y_{N}, Z_{1}, \cdots, Z_{N-1}\right)=\sum_{i=1}^{N} Y_{i}-h\left(Z_{1}, \cdots, Z_{N-1}\right)
$$

where $h$ is an arbitrary function in terms of $Z_{1}, \cdots, Z_{N-1}$. We introduce the field $\sigma$ in the form

$$
\dot{\sigma}=\sum_{i=1}^{N} \beta_{i} \dot{\phi}_{i}, \quad \beta_{i} \equiv \frac{\dot{\phi}_{i}}{\sqrt{\sum_{i=1}^{N} \dot{\phi}_{i}^{2}}} .
$$

The scaling solution (2.23) satisfies

$$
\beta_{i}=\frac{\lambda}{\lambda_{i}}, \quad \text { where } \quad \frac{1}{\lambda^{2}}=\sum_{i=1}^{N} \frac{1}{\lambda_{i}^{2}} .
$$

Then, the scaling solution in the $N$-field system obeys the effective single-field trajectory given by Eq. (2.36).

\section{AUTONOMOUS EQUATIONS AND FIXED POINTS FOR THE MULTI-FIELD SCALING LAGRANGIAN}

In this section we derive the dynamical equations of motion and fixed points for the general Lagrangian (2.21) with $N$ scalar fields. We also take into account a barotropic perfect fluid coupled to the fields $\phi_{i}$ with $Q_{i}$ given in Eq. (2.2).

\section{A. Autonomous equations}

In order to study the autonomous equations of motion, we introduce the following dimensionless variables:

$$
x_{i} \equiv \frac{\dot{\phi}_{i}}{\sqrt{6} H}, \quad y \equiv \frac{e^{-\lambda_{1} \phi_{1} / 2}}{\sqrt{3} H}, \quad(i=1,2, \cdots, N),
$$

and $Z_{i}$ defined in Eq. (2.22) with $i=1,2, \cdots, N-1$. It is convenient to notice the following relations

$$
\begin{aligned}
& X_{i}=3 H^{2} x_{i}^{2}, \quad Y_{i}=x_{i}^{2} / y^{2}, \quad P_{, X_{i}}=g_{, Y_{i}}, \\
& P_{,_{1}}=3 \lambda_{1} H^{2}\left[\sum_{i=1}^{N} x_{i}^{2} g_{, Y_{i}}-g y^{2}-y^{2} \sum_{i=1}^{N-1} \frac{g, Z_{i}}{\lambda_{i+1}}\right], \quad P_{, \phi_{i}}=3 H^{2} y^{2} g_{, Z_{i-1}}, \quad(i \geq 2), \\
& \Omega_{x}=\sum_{i=1}^{N} 2 x_{i}^{2} g_{, Y_{i}}-g y^{2}, \quad w_{x}=\frac{g y^{2}}{\sum_{i=1}^{N} 2 x_{i}^{2} g_{, Y_{i}}-g y^{2}} .
\end{aligned}
$$

From Eq. (3.2) we find

$$
\begin{aligned}
& g y^{2}=\Omega_{x} w_{x} \\
& \sum_{i=1}^{N} 2 x_{i}^{2} g_{, Y_{i}}=\Omega_{x}\left(1+w_{x}\right) .
\end{aligned}
$$


On using Eqs. (2.4)-(2.8), we obtain the following autonomous equations

$$
\begin{aligned}
x_{1}^{\prime}= & \frac{3}{2} x_{1}\left[w_{m}+\left(w_{x}-w_{m}\right) \Omega_{x}-1\right] \\
& -\frac{1}{g_{, Y_{1}}}\left[x_{1} g_{, Y_{1}}^{\prime}-\frac{\sqrt{6}}{2} \lambda_{1}\left\{\sum_{i=1}^{N} x_{i}^{2} g_{, Y_{i}}-g y^{2}-y^{2} \sum_{i=1}^{N-1} \frac{g_{, Z_{i}}}{\lambda_{i+1}}\right\}+\frac{\sqrt{6}}{2} Q_{1}\left(1-\Omega_{x}\right)\right] \\
x_{i}^{\prime}= & \frac{3}{2} x_{i}\left[w_{m}+\left(w_{x}-w_{m}\right) \Omega_{x}-1\right]-\frac{1}{g_{, Y_{i}}}\left[x_{i} g_{, Y_{i}}^{\prime}-\frac{\sqrt{6}}{2} y^{2} g_{, Z_{i-1}}+\frac{\sqrt{6}}{2} Q_{i}\left(1-\Omega_{x}\right)\right], \quad(i=2,3, \cdots, N),(3 . \\
y^{\prime}= & y\left[-\frac{\sqrt{6}}{2} \lambda_{1} x_{1}+\frac{3}{2}\left\{w_{m}+\left(w_{x}-w_{m}\right) \Omega_{x}+1\right\}\right] \\
Z_{i}^{\prime}= & \sqrt{6}\left(x_{i+1}-\frac{\lambda_{1}}{\lambda_{i+1}} x_{1}\right), \quad(i=1,2, \cdots, N-1)
\end{aligned}
$$

This system is described by $2 N$ differential equations of motion. For a given $g\left(Y_{1}, \cdots Y_{N}, Z_{1}, \cdots, Z_{N-1}\right)$, the cosmological evolution is known by solving Eqs. (3.5)-(3.8).

\section{B. Fixed points of assisted inflation and the scaling solution}

Let us search for fixed points with constant values of $x_{i}, y$, and $Z_{i}$. We caution that there are some cases in which $y$ and $Z_{i}$ vary in time, while keeping the variables such as $x_{i}$ and $g y^{2}$ remain constant. We will see such examples in Sec. V.

Since $g_{, Y_{1}}$ and $g_{, Y_{2}}$ are constants, it follows that $g_{, Y_{1}}^{\prime}=0$ and $g_{Y_{2}}^{\prime}=0$. The fixed points relevant to scaling solutions discussed in Sec. II correspond to

$$
y \neq 0
$$

From Eqs. (3.7) and (3.8) we obtain

$$
x_{i}=\frac{\sqrt{6}}{2 \lambda_{i}}\left(1+w_{\mathrm{eff}}\right), \quad \text { for } \quad i=1,2, \cdots, N,
$$

where $w_{\text {eff }}$ is given by Eq. (2.10). Substituting Eq. (3.10) into Eqs. (3.5)-(3.6) and using the relations (3.3)-(3.4), we obtain the following relations

$$
\begin{aligned}
y^{2} \sum_{i=1}^{N-1} \frac{g_{, Z_{i}}}{\lambda_{i+1}} & =\frac{1}{2} \Omega_{x}\left(1-w_{x}\right)-\frac{3 g_{, Y_{1}}}{2 \lambda_{1}^{2}}\left(1-w_{\mathrm{eff}}^{2}\right)-\frac{Q_{1}}{\lambda_{1}}\left(1-\Omega_{x}\right), \\
y^{2} g_{, Z_{i-1}} & =\frac{3 g_{, Y_{i}}}{2 \lambda_{i}}\left(1-w_{\mathrm{eff}}^{2}\right)+Q_{i}\left(1-\Omega_{x}\right), \quad(i=2,3, \cdots, N) .
\end{aligned}
$$

Changing each subscript $i$ in Eq. (3.12) to $i+1$, dividing the resulting equation by $\lambda_{i+1}$, and summing up each equation from $i=1$ to $i=N-1$, it follows that

$$
y^{2} \sum_{i=1}^{N-1} \frac{g, Z_{i}}{\lambda_{i+1}}=\sum_{i=1}^{N-1}\left[\frac{3 g, Y_{i+1}}{2 \lambda_{i+1}^{2}}\left(1-w_{\mathrm{eff}}^{2}\right)+\frac{Q_{i+1}}{\lambda_{i+1}}\left(1-\Omega_{x}\right)\right] .
$$

From Eqs. (3.11) and (3.13) we find

$$
\Omega_{x}\left(1-w_{x}\right)-\left(1-w_{\mathrm{eff}}^{2}\right) \sum_{i=1}^{N} \frac{3 g_{, Y_{i}}}{\lambda_{i}^{2}}=2 q\left(1-\Omega_{x}\right)
$$

where

$$
q \equiv \sum_{i=1}^{N} \frac{Q_{i}}{\lambda_{i}}
$$


Substitution of Eqs. 3.10) into Eq. (3.4) reads

$$
\sum_{i=1}^{N} \frac{3 g_{, Y_{i}}}{\lambda_{i}^{2}}=\frac{\Omega_{x}\left(1+w_{x}\right)}{\left(1+w_{\mathrm{eff}}\right)^{2}}
$$

Plugging Eqs. (3.16) and (2.10) into Eq. (3.14), we obtain

$$
\left(\Omega_{x}-1\right)\left[(1+q)\left(w_{m}-w_{x}\right) \Omega_{x}-q\left(1+w_{m}\right)\right]=0 .
$$

This shows that there exist the following two fixed points:

- Point A: a scalar-field dominated point with

$$
\Omega_{x}=1
$$

- Point B: a scaling solution with

$$
\Omega_{x}=\frac{q\left(1+w_{m}\right)}{\left(w_{m}-w_{x}\right)(1+q)}
$$

In the single-field limit $q \rightarrow Q_{1} / \lambda_{1}$, this recovers the result $\Omega_{x}=Q_{1}\left(1+w_{m}\right) /\left[\left(w_{m}-w_{x}\right)\left(\lambda_{1}+Q_{1}\right)\right]$ derived in Ref. [16].

In the following we discuss the above two fixed points in more detail.

\section{Point A: the scalar-field dominated point}

Substituting $\Omega_{x}=1$ into Eqs. (2.10) and (3.3), it follows that $w_{\text {eff }}=w_{x}=g y^{2}$. On using Eqs. (3.10), (3.16), and (3.12) we obtain the relations

$$
\begin{aligned}
& g=\frac{\sqrt{6} \lambda_{1} x_{1}-3}{3 y^{2}}, \\
& \sum_{i=1}^{N} \frac{g_{, Y_{i}}}{\lambda_{i}^{2}}=\frac{1}{\sqrt{6} \lambda_{1} x_{1}}, \\
& \frac{g_{, Z_{i}}}{g_{, Y_{i+1}}}=\frac{\lambda_{1} x_{1}\left(\sqrt{6}-\lambda_{1} x_{1}\right)}{\lambda_{i+1} y^{2}} .
\end{aligned}
$$

From Eq 3.21 we have

$$
x_{1}=\frac{\lambda^{2}}{\sqrt{6} \lambda_{1}},
$$

where

$$
\frac{1}{\lambda^{2}} \equiv \sum_{i=1}^{N} \frac{g_{, Y_{i}}}{\lambda_{i}^{2}}
$$

Substituting Eq. 3.23) into Eq. 3.20), the effective equation of state $w_{\text {eff }}=g y^{2}$ can be expressed as

$$
w_{\text {eff }}=-1+\frac{\lambda^{2}}{3} .
$$

The cosmic acceleration occurs for $\lambda^{2}<2$. Even if each field does not lead to the cosmic acceleration, the presence of multiple fields can do so by reducing the value of $\lambda^{2}$ relative to $\lambda_{i}^{2} / g_{, Y_{i}}$. This is the phenomenon of assisted inflation, which is known to occur for canonical scalar fields with exponential potentials [21]. The above argument shows that the assisted inflationary mechanism is also present for the general multi-field scaling Lagrangian (2.21). For a given function $g$ we can solve Eqs. (3.20)-(3.22) for $x_{i}, y$, and $Z_{i}$ by noting the relations $Y_{i}=x_{i}^{2} / y^{2}$ and $Y_{j}=Y_{i} \lambda_{i}^{2} / \lambda_{j}^{2}$. 
For example, let us consider the two-field model

$$
g\left(Y_{1}, Y_{2}, Z_{1}\right)=Y_{1}+Y_{2}-V_{1}-V_{2} e^{-\lambda_{2} Z_{1}},
$$

which corresponds to $\mu_{1}=0$ and $\mu_{2}=-\lambda_{2}$ in Eq. (2.26). This model is characterized by two canonical fields with exponential potentials: $P=X_{1}+X_{2}-V_{1} e^{-\lambda_{1} \phi_{1}}-V_{2} e^{-\lambda_{2} \phi_{2}}$ [21]. Solving Eqs. (3.20)-(3.22) in this case, we obtain $x_{1}=\lambda^{2} /\left(\sqrt{6} \lambda_{1}\right)$ and

$$
y^{2}=\frac{1}{V_{1}}\left(1-\frac{\lambda^{2}}{6}\right) \frac{\lambda^{2}}{\lambda_{1}^{2}}, \quad e^{-\lambda_{2} Z_{1}}=\frac{\lambda_{1}^{2}}{\lambda_{2}^{2}} \frac{V_{1}}{V_{2}},
$$

where $1 / \lambda^{2}=1 / \lambda_{1}^{2}+1 / \lambda_{2}^{2}$. The density parameters of the fields $\phi_{1}$ and $\phi_{2}$ are given by $\Omega_{x_{1}}=x_{1}^{2}+V_{1} y^{2}=\lambda^{2} / \lambda_{1}^{2}$ and $\Omega_{x_{2}}=x_{2}^{2}+V_{2} y^{2} e^{-\lambda_{2} Z_{1}}=\lambda^{2} / \lambda_{2}^{2}$, respectively, so that $\Omega_{x_{1}} / \Omega_{x_{2}}=\lambda_{2}^{2} / \lambda_{1}^{2}$. The field with a smaller value of $\lambda_{i}$ has a lager energy fraction than that of another field.

\section{Point B: the scaling solution}

Substituting Eq. (3.19) into Eq. (2.10), the effective equation of state for the scaling solution is given by

$$
w_{\text {eff }}=\frac{w_{m}-q}{1+q},
$$

where $q$ is defined by Eq. (3.15). For non-relativistic matter $\left(w_{m}=0\right)$ the presence of the couplings $Q_{i}$ can lead to cosmic acceleration for $q>1 / 2$. From Eqs. (3.10), (3.16) and (3.3), we obtain the following relations

$$
\begin{aligned}
x_{1} & =\frac{\sqrt{6}}{2 \lambda_{1}} \frac{1+w_{m}}{1+q}, \\
\frac{1}{\lambda^{2}} & =\frac{\Omega_{x}\left(1+w_{x}\right)(1+q)^{2}}{3\left(1+w_{m}\right)^{2}}, \\
g y^{2} & =\frac{w_{x}\left(1+w_{m}\right) q}{\left(w_{m}-w_{x}\right)(1+q)},
\end{aligned}
$$

where $\lambda^{2}$ is defined in Eq. (3.24). Using Eqs. (3.19) and (3.30), $\Omega_{x}$ and $w_{x}$ can be written as

$$
\begin{aligned}
& \Omega_{x}=\frac{3\left(1+w_{m}\right)+q(1+q) \lambda^{2}}{(1+q)^{2} \lambda^{2}}, \\
& w_{x}=\frac{3 w_{m}\left(1+w_{m}\right)-q(1+q) \lambda^{2}}{3\left(1+w_{m}\right)+q(1+q) \lambda^{2}} .
\end{aligned}
$$

In the $q \rightarrow 0$ limit we have $\Omega_{x}=3\left(1+w_{m}\right) / \lambda^{2}$ and $w_{\text {eff }}=w_{x}=w_{m}$, so the energy density of the scalar fields scale as that of the background fluid. For the physically meaningful scaling solution characterized by $\Omega_{x}<1$, we require that $\lambda^{2}>3\left(1+w_{m}\right)$. This condition is incompatible with that for the cosmic acceleration of the fixed point A $\left(\lambda^{2}<2\right)$. However, if $\lambda_{1}^{2}$ is larger than the order of 1 and $\lambda_{2}^{2}$ is smaller than $O(1)$, there is a possibility that the field $\phi_{1}$ is in the scaling regime during the radiation and matter eras and that the field $\phi_{2}$ leads to the acceleration of the Universe at late times. We will discuss these cases in Sec. $\mathbf{V}$.

In the presence of the couplings $Q_{i}$, we also have another possibility to give rise to the accelerated expansion by the scaling fixed point B with $\Omega_{x} \simeq 0.7$ for $q>1 / 2$. In this case the standard matter era is also replaced by another solution (fixed point $\mathrm{C}$ discussed below). In the single-field scenario it was shown that the scaling accelerated solution $\mathrm{B}$ preceded by the modified matter era is difficult to be realized for a vast class of scaling models [17]. In Sec. $\nabla$ we mention this possibility for the two-field model (2.26).

Provided that the function $g$ is given, we can solve Eqs. (3.31) and (3.12) for $y$ and $Z_{1}$. For the model (3.26) with $w_{m}=0$, it follows that

$$
\begin{aligned}
y^{2} & =\frac{2 Q_{2} \lambda_{2}\left(3+Q_{1} \lambda_{1}\right)+\left[3+2 Q_{1}\left(Q_{1}+\lambda_{1}\right)\right] \lambda_{2}^{2}-6 Q_{1} \lambda_{1}}{2 V_{1}\left[Q_{1} \lambda_{2}+\left(Q_{2}+\lambda_{2}\right) \lambda_{1}\right]^{2}}, \\
e^{\lambda_{2} Z_{1}} & =\frac{2 Q_{2} \lambda_{2}\left(3+Q_{1} \lambda_{1}\right)+\left[3+2 Q_{1}\left(Q_{1}+\lambda_{1}\right)\right] \lambda_{2}^{2}-6 Q_{1} \lambda_{1}}{2 Q_{1} \lambda_{1}\left(3+Q_{2} \lambda_{2}\right)+\left[3+2 Q_{2}\left(Q_{2}+\lambda_{2}\right)\right] \lambda_{1}^{2}-6 Q_{2} \lambda_{2}} \frac{V_{2}}{V_{1}},
\end{aligned}
$$

and $\Omega_{x}$ and $w_{x}$ given by Eqs. (3.32) and (3.33) with $g_{, Y_{1}}=g_{, Y_{2}}=1$ and $w_{m}=0$. 


\section{Kinetic fixed points}

The kinetic fixed points correspond to non-zero values of $x_{i}(i=1,2, \cdots, N)$ with

$$
y=0 .
$$

Since the variables $Y_{i}=x_{i}^{2} / y^{2}$ diverge in this case, this restricts the Lagrangian (2.21) to specific forms. Since the pressure $P$ is proportional to $y^{2} g$, the function $g$ can contain the terms linear in $Y_{i}$, but not the terms like $Y_{i}^{n}(n \geq 2)$. Let us then consider the function of the form

$$
g=\sum_{i=1}^{N} c_{i} Y_{i}+\sum_{i=1}^{N} \sum_{n>0} c_{-n}^{(i)} Y_{i}^{-n}-h\left(Z_{1}, \cdots, Z_{N-1}\right),
$$

where $c_{i}$ and $c_{-n}^{(i)}$ are constants with positive integer $n$, and $h$ is a finite function with respect to $Z_{1}, \cdots, Z_{N-1}$. In order to avoid the appearance of ghosts, we focus on the case $c_{i}>0$. For the choice (3.37) we have

$$
g y^{2}(y \rightarrow 0)=\sum_{i=1}^{N} c_{i} x_{i}^{2}, \quad g_{, Y_{i}}(y \rightarrow 0)=c_{i} .
$$

We also consider the case in which $g_{, Z_{i}}$ is finite, such that $y^{2} g_{, Z, i} \rightarrow 0$ for $y \rightarrow 0$. Equations (3.5) and (3.6) read

$$
x_{i}^{\prime}=\frac{3}{2} x_{i}\left[w_{m}+\left(w_{x}-w_{m}\right) \Omega_{x}-1\right]-\frac{\sqrt{6}}{2 c_{i}} Q_{i}\left(1-\Omega_{x}\right), \quad(i=1,2, \cdots, N) .
$$

From Eqs. (3.3), (3.4) and (3.38), we obtain $\Omega_{x}\left(w_{x}-1\right)=0$, that is, $\Omega_{x}=0$ or $w_{x}=1$. When $\Omega_{x}=0$ we have $\sum_{i=1}^{N} c_{i} x_{i}^{2}=0$ and hence $x_{i}=0$ for $c_{i}>0(i=1,2, \cdots, N)$. From Eq. (3.39) this can be realized only for $Q_{i}=0$. When $w_{m}=0$, this corresponds to the standard matter-dominated epoch.

In the rest of this section we study the case

$$
w_{x}=1
$$

Then, Eq. (3.39) reads

$$
x_{i}^{\prime}=\frac{1}{2}\left[3\left(w_{m}-1\right) x_{i}-\frac{\sqrt{6} Q_{i}}{c_{i}}\right]\left(1-\Omega_{x}\right) .
$$

There are two qualitatively different fixed points:

- Point C: a $\phi$-matter-dominated era ( $\phi \mathrm{MDE})$ [31] with

$$
x_{i}=\frac{\sqrt{6} Q_{i}}{3 c_{i}\left(w_{m}-1\right)}, \quad(i=1,2 \cdots, N) .
$$

- Point D: a purely kinetic solution with

$$
\Omega_{x}=1
$$

\section{Point $C$ : the $\phi M D E$}

Substituting the solutions (3.42) into $\Omega_{x}=\sum_{i=1}^{N} c_{i} x_{i}^{2}$, we obtain

$$
\Omega_{x}=\frac{2}{3\left(w_{m}-1\right)^{2}} \sum_{i=1}^{N} \frac{Q_{i}^{2}}{c_{i}} .
$$

This corresponds to the scaling solution appearing in the presence of the couplings $Q_{i}$. The effective equation of state is given by

$$
w_{\mathrm{eff}}=w_{m}+\frac{2}{3\left(1-w_{m}\right)} \sum_{i=1}^{N} \frac{Q_{i}^{2}}{c_{i}} .
$$




\begin{tabular}{|c|c|c|c|c|}
\hline & $x_{1}$ & $\Omega_{x}$ & $w_{\text {eff }}$ & $w_{x}$ \\
\hline \hline $\mathrm{A}$ & $\frac{\lambda^{2}}{\sqrt{6} \lambda_{1}}$ & 1 & $-1+\frac{\lambda^{2}}{3}$ & $-1+\frac{\lambda^{2}}{3}$ \\
\hline $\mathrm{B}$ & $\frac{\sqrt{6}\left(1+w_{m}\right)}{2 \lambda_{1}(1+q)}$ & $\frac{3\left(1+w_{m}\right)+q(1+q) \lambda^{2}}{(1+q)^{2} \lambda^{2}}$ & $\frac{w_{m}-q}{1+q}$ & $\frac{3 w_{m}\left(1+w_{m}\right)-q(1+q) \lambda^{2}}{3\left(1+w_{m}\right)+q(1+q) \lambda^{2}}$ \\
\hline $\mathrm{C}$ & $\frac{\sqrt{6} Q_{1}}{3 c_{1}\left(w_{m}-1\right)}$ & $\frac{2}{3\left(w_{m}-1\right)^{2}} \sum_{i=1}^{N} \frac{Q_{i}^{2}}{c_{i}}$ & $w_{m}+\frac{2}{3\left(1-w_{m}\right)} \sum_{i=1}^{N} \frac{Q_{i}^{2}}{c_{i}}$ & 1 \\
\hline $\mathrm{D}$ & $\sum_{i=1}^{N} c_{i} x_{i}^{2}=1$ & 1 & 1 & 1 \\
\hline
\end{tabular}

Table I: The properties of four fixed points A, B, C, and D, where $\lambda, q$ and $c_{i}$ are defined in Eqs. (3.24), (3.15) and (3.37), respectively. The points $\mathrm{A}$ and $\mathrm{B}$ exist for the general $N$-field Lagrangian (2.21), whereas the points $\mathrm{C}$ and D arise for the specific Lagrangian $P=e^{-\lambda_{1} \phi_{1}} g$ with $g$ given by Eq. (3.37).

When $w_{m}=0$, it follows that $w_{\text {eff }}=\Omega_{x}=(2 / 3) \sum_{i=1}^{N} Q_{i}^{2} / c_{i}$. For $Q_{i} \neq 0$ the $\phi$ MDE can replace the standard matter era characterized by $w_{\text {eff }}=\Omega_{x}=0$. For the consistency with observations we require that $w_{\text {eff }} \ll 1$, by which the couplings $Q_{i}$ are bounded from above [39]. The $\phi \mathrm{MDE}$ should be followed by some fixed points like $\mathrm{A}$ and $\mathrm{B}$ to enter the regime of cosmic acceleration.

For the derivation of the $\phi \mathrm{MDE}$ discussed above the constancy of the terms $Z_{i}$ is not actually required, so the relation $x_{i}=\lambda_{1} x_{1} / \lambda_{i}$ does not need to hold. This property is different from that discussed for the scaling solution in Sec. III.

\section{Point D: the purely kinetic solution}

Substituting $\Omega_{x}=1$ and $w_{x}=1$ into Eq. (3.4), we obtain

$$
\sum_{i=1}^{N} c_{i} x_{i}^{2}=1 .
$$

If the variables $Z_{i}$ are constant, there is the relation $x_{i}=\lambda_{1} x_{1} / \lambda_{i}$ and hence $x_{1}$ is known from Eq. (3.46). However, this condition is not mandatory because $Z_{i}$ can vary in time. From Eqs. (2.10) and (3.40) we have

$$
w_{\mathrm{eff}}=w_{x}=1 .
$$

This solution can be used neither for the matter era nor for the epoch of cosmic acceleration.

The four fixed points are summarized in Table 【

\section{STABILITY OF THE FIXED POINTS A AND B}

In this section we study the stability of the fixed points A and B in the presence of a barotropic perfect fluid with the equation of state $w_{m}$. We consider the two-field model characterized by the Lagrangian

$$
P=e^{-\lambda_{1} \phi_{1}} g\left(Y_{1}, Y_{2}, Z_{1}\right) .
$$

For the point A it is possible to carry out the general analysis without restricting the functional form of $g\left(Y_{1}, Y_{2}, Z_{1}\right)$. Since the general stability analysis for the point B is very complicated, we focus on a simpler model with two canonical fields. We also interpret the stability conditions of the scaling solution B as a geometric perspective in the two-field space. For the stability of the other fixed points like $\mathrm{C}$ and D, we will discuss it in Sec. $\mathrm{V}$ for a more concrete two-field model.

\section{A. Fixed point A}

Let us first study the stability of the fixed point A characterized by the conditions (3.20)-(3.25). Taking the $\mathcal{N}$-derivative of the field density parameter $\Omega_{x}=2\left(x_{1}^{2} g_{, Y_{1}}+x_{2}^{2} g_{, Y_{2}}\right)-g y^{2}$ and using Eqs. (3.5)-(3.7), we obtain

$$
\Omega_{x}^{\prime}=\left(\Omega_{x}-1\right)\left[3\left(w_{x}-w_{m}\right) \Omega_{x}+\sqrt{6}\left(Q_{1} x_{1}+Q_{2} x_{2}\right)\right] .
$$


Perturbing this equation at linear order and denoting the perturbed quantities like $\delta \Omega_{x}$, we have

$$
\delta \Omega_{x}^{\prime}=\left[3\left(w_{x}-w_{m}\right) \Omega_{x}+\sqrt{6}\left(Q_{1} x_{1}+Q_{2} x_{2}\right)\right] \delta \Omega_{x}+\left(\Omega_{x}-1\right)\left[3 y^{2} \delta g+6 g y \delta y-3 w_{m} \delta \Omega_{x}+\sqrt{6}\left(Q_{1} \delta x_{1}+Q_{2} \delta x_{2}\right)\right] .
$$

Since $\Omega_{x}=1, w_{x}=-1+\lambda^{2} / 3$, and $x_{i}=\lambda^{2} /\left(\sqrt{6} \lambda_{i}\right)$ for the point A, it follows that

$$
\delta \Omega_{x}^{\prime}=\left[\lambda^{2}(1+q)-3\left(1+w_{m}\right)\right] \delta \Omega_{x} .
$$

Hence the point $\mathrm{A}$ is stable in the $\Omega_{x}$ direction under the condition

$$
\lambda^{2}(1+q)<3\left(1+w_{m}\right) .
$$

Once this condition is satisfied, then we need to study the behavior of the other variables. The presence of $\delta \Omega_{x}$ acts as an explicitly time-dependent inhomogeneous term in the other perturbed equations of motion. Therefore, in order to study the stability of the other variables, we will consider only the homogeneous contribution, and we will set $\delta \Omega_{x}$ to vanish in the remaining equations.

Let us consider the quantities $Y_{1}, Y_{2}$, and $Z_{1}$ as other dynamical variables. Then we can express both $\delta Y_{1}$ and $\delta Y_{2}$ in terms of $\delta Z_{1}$ and its derivatives. Finally, we obtain a third-order differential equation for $\delta Z_{1}$. On looking for a solution of the kind $\delta Z_{1} \propto e^{\Gamma \mathcal{N}}$, we find that $\Gamma$ satisfies a cubic equation, which can be factorized as

$$
\left(2 \Gamma+6-\lambda^{2}\right)\left(\alpha \Gamma^{2}+\beta \Gamma+\delta\right)=0,
$$

where

$$
\begin{aligned}
& \alpha=2 \lambda_{1}^{2} \lambda_{2}^{2} Y_{1}\left[2 \lambda_{1}^{4} \lambda^{2} Y_{1}\left(g_{, Y_{1}} g_{, Y_{2} Y_{2}}-2 Y_{1} g_{, Y_{1} Y_{2}}^{2}+2 Y_{1} g_{, Y_{1} Y_{1}} g_{, Y_{2} Y_{2}}\right)+\lambda_{1}^{2} \lambda_{2}^{2} \lambda^{2} g_{, Y_{2}}\left(g_{, Y_{1}}+2 Y_{1} g_{, Y_{1} Y_{1}}\right)\right] \text {, } \\
& \beta=\frac{1}{2}\left(6-\lambda^{2}\right) \alpha, \\
& \delta=\lambda_{2}^{2}\left[Y_{1} \lambda^{2} \lambda_{1}^{2} g_{, Y_{1} Y_{2}}\left\{Y_{1} \lambda_{2}\left[2 \lambda^{2}\left(\lambda^{2}-6\right) \lambda_{1}^{2} g_{, Y_{1} Z_{1}}-\lambda_{2}\left(\lambda^{2} g_{, Y_{1}}-\lambda_{1}^{2}\right)\left(12 \lambda^{2} g_{, Y_{1}}+\left(\lambda^{2}-18\right) \lambda_{1}^{2}\right)\right]-4 \lambda^{4} \lambda_{1}^{2} g_{, Z_{1}} Z_{1}\right\}\right. \\
& +\lambda_{2}^{2}\left\{2 Y_{1} \lambda_{1}^{2} \lambda^{6}\left(g_{, Y_{1} Z_{1}}^{2}-g_{, Y_{1} Y_{1}} g_{, Z_{1} Z_{1}}\right)-3 Y_{1}\left(\lambda^{2}-2\right) \lambda_{1}^{2} \lambda_{2} \lambda^{2} g_{, Y_{1} Z_{1}}\left(\lambda^{2} g_{, Y_{1}}-\lambda_{1}^{2}\right)\right. \\
& \left.\left.+Y_{1} \lambda_{2}^{2}\left(\lambda_{1}^{2}-\lambda^{2} g_{, Y_{1}}\right)^{2}\left[\left(\lambda^{2}-6\right) \lambda_{1}^{2}-6 Y_{1} \lambda^{2} g_{, Y_{1} Y_{1}}\right]-\lambda_{1}^{4} \lambda^{4} g_{, Z_{1} Z_{1}}\right\}\right]+2 Y_{1} \lambda^{6} \lambda_{1}^{6} g_{, Y_{2} Z_{1}}^{2} \\
& +Y_{1} \lambda^{4} \lambda_{2} \lambda_{1}^{4} g_{, Y_{2} Z_{1}}\left[4 \lambda_{2} \lambda^{2} g_{, Y_{1} Z_{1}}-2 Y_{1}\left(\lambda^{2}-6\right) \lambda_{1}^{2} g_{, Y_{1} Y_{2}}+\lambda_{2}^{2}\left(2 \lambda_{1}^{2}-3\left(\lambda^{2}-2\right) g_{, Y_{1}}-2 Y_{1}\left(\lambda^{2}-6\right) g_{, Y_{1} Y_{1}}\right)\right] \\
& +Y_{1} \lambda^{2} \lambda_{1}^{4} g_{, Y_{2} Y_{2}}\left\{Y_{1} \lambda_{2}\left[2 \lambda^{2}\left(\lambda^{2}-6\right) \lambda_{1}^{2} g_{, Y_{1} Z_{1}}-\lambda_{2}\left(\lambda^{2} g_{, Y_{1}}-\lambda_{1}^{2}\right)\left(6 \lambda^{2} g_{, Y_{1}}+\left(\lambda^{2}-12\right) \lambda_{1}^{2}\right)\right]-2 \lambda^{4} \lambda_{1}^{2} g_{, Z_{1} Z_{1}}\right\} .
\end{aligned}
$$

The three solutions to Eq. (4.6) are the following

$$
\begin{aligned}
\Gamma_{1} & =-\frac{1}{2}\left(6-\lambda^{2}\right), \\
\Gamma_{2,3} & =-\frac{1}{4}\left(6-\lambda^{2}\right)\left[1 \pm \sqrt{1-\frac{4 \alpha \delta}{\beta^{2}}}\right] .
\end{aligned}
$$

The stability of the point $A$ demands that $\Gamma_{1}, \Gamma_{2}, \Gamma_{3}$ are negative, or if they are imaginary, they have negative real parts. This requires the following conditions

$$
\begin{aligned}
& \lambda^{2}<6, \\
& \alpha \delta>0 .
\end{aligned}
$$

If the fixed point $\mathrm{A}$ is responsible for cosmic acceleration, then $w_{\text {eff }}=-1+\lambda^{2} / 3<-1 / 3$ and $\lambda^{2}<2$. In this case the condition (4.12) is automatically satisfied. In summary, the point A is stable under the conditions (4.5), 4.12), and (4.13).

For concreteness, we study the model

$$
g\left(Y_{1}, Y_{2}, Z_{1}\right)=Y_{1}+Y_{2}-h\left(Z_{1}\right),
$$

in which case the field potential is given by Eq. (2.32). For the positivity of the potential we demand the condition $h\left(Z_{1}\right)>0$. From Eq. (3.20) we have that $g y^{2}=x_{1}^{2}+x_{2}^{2}-y^{2} h=-1+\sqrt{6} \lambda_{1} x_{1} / 3$. Substituting $x_{1}=\lambda^{2} /\left(\sqrt{6} \lambda_{1}\right)$ and $x_{2}=\lambda^{2} /\left(\sqrt{6} \lambda_{2}\right)$ into this relation, it follows that

$$
y^{2}=\frac{1}{h}\left(1-\frac{\lambda^{2}}{6}\right), \quad Y_{1}=\frac{\lambda^{4}}{\lambda_{1}^{2}\left(6-\lambda^{2}\right)} h .
$$


Combining Eq. (3.22) with the first of Eq. (4.15), we obtain

$$
h, Z_{1}=-\frac{\lambda^{2}}{\lambda_{2}} h .
$$

The quantities $\alpha$ and $\delta$ read

$$
\alpha=\frac{2 \lambda_{1}^{2} \lambda_{2}^{4} \lambda^{6}}{6-\lambda^{2}} h, \quad \delta=\left(\lambda_{1} \lambda_{2} \lambda\right)^{4}\left(h, Z_{1} Z_{1}-\frac{\lambda^{4}}{\lambda_{2}^{2}} h\right) .
$$

Since $\alpha>0$, the condition (4.13) translates to $\delta>0$, that is

$$
h, Z_{1} Z_{1}>\frac{\lambda^{4}}{\lambda_{2}^{2}} h .
$$

The stability of the fixed point A with cosmic acceleration $\left(\lambda^{2}<2\right)$ is ensured under the conditions (4.5) and (4.18) together with the additional constraint (4.16).

As an example, let us consider the model (2.26), i.e.,

$$
h\left(Z_{1}\right)=V_{1} e^{\mu_{1} Z_{1}}+V_{2} e^{\mu_{2} Z_{1}} .
$$

In this case, Eqs. (4.16) and (4.18) translate to

$$
\begin{aligned}
& \frac{V_{2}}{V_{1}}=-e^{\left(\mu_{1}-\mu_{2}\right) Z_{1}} \frac{\mu_{1}+\lambda^{2} / \lambda_{2}}{\mu_{2}+\lambda^{2} / \lambda_{2}}, \\
& V_{1}\left(\mu_{1}+\lambda^{2} / \lambda_{2}\right)\left(\mu_{1}-\mu_{2}\right)>0,
\end{aligned}
$$

respectively. The positivity of the potential $\left(V_{1}>0\right.$ and $\left.V_{2}>0\right)$ in Eq. 4.20) requires that

$$
\left(\mu_{1}+\lambda^{2} / \lambda_{2}\right)\left(\mu_{2}+\lambda^{2} / \lambda_{2}\right)<0,
$$

under which (4.21) is automatically satisfied. The condition (4.22), together with (4.5), ensures the stability of the fixed point A.

\section{B. Fixed point $B$}

We can proceed to study the stability of the point B along the same lines shown in Sec. IVA. In this case, however, the equation of motion for the variable $\delta \Omega_{x}$ does not decouple from the other variables. This makes the analysis more cumbersome. Nonetheless, since we are dealing with linear differential equations with constant coefficients, the algorithm for the solution is straightforward. Namely, we can look for solutions of the kind $\delta Y_{1}=A_{1} \exp (\Gamma \mathcal{N})$, $\delta Y_{2}=A_{2} \exp (\Gamma \mathcal{N}), \delta Z_{1}=A_{3} \exp (\Gamma \mathcal{N})$, and $\delta \Omega_{x}=A_{4} \exp (\Gamma \mathcal{N})$. Then we can use three of the four differential equations to write e.g., $A_{1,2,3}$ in terms of $A_{3}$ and $\Gamma$. The last available equation transforms into a quartic algebraic equation for the variable $\Gamma$. Although the general solutions can be given, they are too complicated to be written here.

The analysis can be simplified by specifying the models. In the following, let us focus on the model

$$
g\left(Y_{1}, Y_{2}, Z_{1}\right)=Y_{1}+Y_{2}-h\left(Z_{1}\right), \quad \text { with } \quad Q_{i}=0 \quad(i=1,2) .
$$

We perturb the autonomous equations of motion about the scaling solution B characterized by Eqs. (3.28)-(3.33). Since the field density parameter in Eq. (3.32) is given by $\Omega_{x}=x_{1}^{2}+x_{2}^{2}+h y^{2}$ with $x_{1}=\sqrt{6}\left(1+w_{m}\right) /\left(2 \lambda_{1}\right)$ and $x_{2}=\sqrt{6}\left(1+w_{m}\right) /\left(2 \lambda_{2}\right)$, we obtain

$$
y^{2}=\frac{3\left(1-w_{m}^{2}\right)}{2 \lambda^{2}} \frac{1}{h}
$$

From Eq. (3.12) there is also the following relation

$$
h_{, Z_{1}}=-\frac{\lambda^{2}}{\lambda_{2}} h .
$$


The linear perturbations $\delta x_{1}, \delta x_{2}, \delta y$, and $\delta Z_{1}$ about the scaling fixed point $\mathrm{B}$ obey the equations of motion

$$
{ }^{t}\left(\delta x_{1}^{\prime}, \delta x_{2}^{\prime}, \delta y^{\prime}, \delta Z_{1}^{\prime}\right)=\mathcal{M}^{t}\left(\delta x_{1}, \delta x_{2}, \delta y, \delta Z_{1}\right),
$$

where $\mathcal{M}$ is a $4 \times 4$ matrix. The four eigenvalues of the matrix $\mathcal{M}$ are given by

$$
\begin{aligned}
& \gamma_{1,2}=-\frac{3}{4}\left(1-w_{m}\right)\left[1 \pm \sqrt{1-\frac{8\left(1+w_{m}\right)\left[\lambda^{2}-3\left(1+w_{m}\right)\right]}{\lambda^{2}\left(1-w_{m}\right)}}\right] \\
& \gamma_{3,4}=-\frac{3}{4}\left(1-w_{m}\right)\left[1 \pm \sqrt{1-\frac{8 \lambda_{1}^{2}\left(1+w_{m}\right)}{\lambda^{4}\left(1-w_{m}\right) h}\left(h_{, Z_{1} Z_{1}}-\frac{\lambda^{4}}{\lambda_{2}^{2}} h\right)} .\right.
\end{aligned}
$$

For $0 \leq w_{m}<1$ the scaling solution $\mathrm{B}$ is stable provided that $\gamma_{1,2,3,4}$ are negative or have negative real parts, that is

$$
\begin{aligned}
& \lambda^{2}>3\left(1+w_{m}\right), \\
& h_{, Z_{1} Z_{1}}>\frac{\lambda^{4}}{\lambda_{2}^{2}} h .
\end{aligned}
$$

The conditions (4.30) and (4.25) are exactly the same as Eqs. (4.18) and (4.16) derived for the stability of the fixed point A with cosmic acceleration. The difference appears for another condition (4.29), whose inequality is opposite to the stability condition (4.5) for the point A with $q=0$. This means that, as far as the model (4.23) is concerned, the scaling solution B is stable (unstable) when the fixed point A with cosmic acceleration is unstable (stable).

It is instructive to understand the meaning of the stability conditions (4.29) and (4.30) as well as the additional constraint (4.25). In doing so, we perform the field rotation analogous to (2.34):

$$
\begin{aligned}
& \phi_{1}=(\cos \theta) \sigma-(\sin \theta) s, \\
& \phi_{2}=(\sin \theta) \sigma+(\cos \theta) s,
\end{aligned}
$$

where $\theta$ is a constant satisfying $\cos \theta=\lambda / \lambda_{1}$ and $\sin \theta=\lambda / \lambda_{2}$. Then the potential (2.32) transforms as

$$
V(\sigma, s)=e^{-\lambda \sigma+\left(\lambda^{2} / \lambda_{2}\right) Z_{1}} h\left(Z_{1}\right), \quad \text { where } \quad Z_{1}=\frac{\lambda_{1}}{\lambda} s .
$$

The field $\sigma$ is transverse to the scaling solution (2.23), whereas the field $s$, or equivalently to $Z_{1}$, points to a direction orthogonal to $\sigma$. The scaling solution satisfies the condition $V_{s}=0$, that is

$$
h_{, Z_{1}}=-\frac{\lambda^{2}}{\lambda_{2}} h
$$

which is equivalent to (4.25). On using this condition, the second derivative of $V$ with respect to $s$ reads

$$
V_{, s s}=\frac{\lambda_{1}^{2}}{\lambda^{2}} e^{-\lambda \sigma+\left(\lambda^{2} / \lambda_{2}\right) Z_{1}}\left(h_{, Z_{1} Z_{1}}-\frac{\lambda^{4}}{\lambda_{2}^{2}} h\right) .
$$

The stability along the direction of the field $s$ demands that $V_{\text {,ss }}>0$, i.e.,

$$
h_{, Z_{1} Z_{1}}>\frac{\lambda^{4}}{\lambda_{2}^{2}} h
$$

which matches with the condition (4.30). Now the scaling solution can be described by an effective single field $\sigma$ having a stability to the orthogonal direction. From Eq. 4.33 the potential is proportional to the exponential term $e^{-\lambda \sigma}$ along the $\sigma$ direction. Hence the stability of the fixed point B in the presence of a barotropic perfect fluid is the same as that derived in Refs. [7, 16] for the single-field exponential potential, that is,

$$
\lambda^{2}>3\left(1+w_{m}\right)
$$

which is the same as another stability condition (4.29). The above discussion explains the physical meaning of the stability conditions (4.29) and (4.30).

If we choose the function $h\left(Z_{1}\right)$ of the form (4.19), Eqs. (4.34) and (4.36) give rise to the same conditions as Eqs. (4.20) and (4.21), respectively. 


\section{CONCRETE MODEL}

In this section we study the cosmology of the two-field model described by the Lagrangian (2.27) with (2.28), that is

$$
P=X_{1}+X_{2}-V_{1} e^{-\lambda_{1}\left(1+\mu_{1} / \lambda_{2}\right) \phi_{1}+\mu_{1} \phi_{2}}-V_{2} e^{-\lambda_{1}\left(1+\mu_{2} / \lambda_{2}\right) \phi_{1}+\mu_{2} \phi_{2}} .
$$

This corresponds to the Lagrangian $P=e^{-\lambda_{1} \phi_{1}} g\left(Y_{1}, Y_{2}, Z_{1}\right)$ with the $g$ given by Eq. (2.26). In addition to nonrelativistic matter with the equation of state $w_{m}=0$, we take into account the radiation with the energy density $\rho_{r}$ satisfying the continuity equation $\dot{\rho}_{r}+4 H \rho_{r}=0$. Instead of $Z_{1}$ and $y$ defined in Eqs. (2.22) and (3.1), we introduce the following quantities

$$
y_{1} \equiv \sqrt{V_{1}} e^{\mu_{1} Z_{1} / 2} y, \quad y_{2} \equiv \sqrt{V_{2}} e^{\mu_{2} Z_{1} / 2} y,
$$

whose squares can be written as

$$
y_{1}^{2}=\frac{V_{1}}{3 H^{2}} e^{-\lambda_{1}\left(1+\mu_{1} / \lambda_{2}\right) \phi_{1}+\mu_{1} \phi_{2}}, \quad y_{2}^{2}=\frac{V_{2}}{3 H^{2}} e^{-\lambda_{1}\left(1+\mu_{2} / \lambda_{2}\right) \phi_{1}+\mu_{2} \phi_{2}} .
$$

These quantities are related to the potential energies of the third and fourth terms on the right-hand side (rhs) of Eq. (5.1) respectively, whereas $x_{1}^{2}$ and $x_{2}^{2}$ are associated with the kinetic energies of the fields $\phi_{1}$ and $\phi_{2}$ respectively. Several quantities appearing in Eqs. (3.5)-(3.8) can be expressed as

$$
\Omega_{x}=x_{1}^{2}+x_{2}^{2}+y_{1}^{2}+y_{2}^{2}, \quad \Omega_{x} w_{x}=x_{1}^{2}+x_{2}^{2}-y_{1}^{2}-y_{2}^{2},
$$

and $g_{, Z_{1}} y^{2}=-\mu_{1} y_{1}^{2}-\mu_{2} y_{2}^{2}$.

Defining the density parameter of the radiation as $\Omega_{r}=\rho_{r} /\left(3 H^{2}\right)$, we obtain the following autonomous equations of motion

$$
\begin{aligned}
x_{1}^{\prime} & =\frac{1}{2} x_{1}\left(3 \Omega_{x} w_{x}-3+\Omega_{r}\right)+\frac{\sqrt{6}}{2} \lambda_{1}\left[y_{1}^{2}+y_{2}^{2}+\frac{1}{\lambda_{2}}\left(\mu_{1} y_{1}^{2}+\mu_{2} y_{2}^{2}\right)\right]-\frac{\sqrt{6}}{2} Q_{1} \Omega_{m}, \\
x_{2}^{\prime} & =\frac{1}{2} x_{2}\left(3 \Omega_{x} w_{x}-3+\Omega_{r}\right)-\frac{\sqrt{6}}{2}\left(\mu_{1} y_{1}^{2}+\mu_{2} y_{2}^{2}\right)-\frac{\sqrt{6}}{2} Q_{2} \Omega_{m}, \\
y_{1}^{\prime} & =\frac{y_{1}}{2}\left[\sqrt{6} \mu_{1}\left(x_{2}-\frac{\lambda_{1}}{\lambda_{2}} x_{1}\right)+3 \Omega_{x} w_{x}+3+\Omega_{r}-\sqrt{6} \lambda_{1} x_{1}\right], \\
y_{2}^{\prime} & =\frac{y_{2}}{2}\left[\sqrt{6} \mu_{2}\left(x_{2}-\frac{\lambda_{1}}{\lambda_{2}} x_{1}\right)+3 \Omega_{x} w_{x}+3+\Omega_{r}-\sqrt{6} \lambda_{1} x_{1}\right], \\
\Omega_{r}^{\prime} & =\Omega_{r}\left(3 \Omega_{x} w_{x}-1+\Omega_{r}\right)
\end{aligned}
$$

where the density parameter of non-relativistic matter is given by

$$
\Omega_{m}=1-\Omega_{x}-\Omega_{r} .
$$

The effective equation of state (2.9) reads

$$
w_{\mathrm{eff}}=\Omega_{x} w_{x}+\frac{1}{3} \Omega_{r}
$$

In the following we first study the cosmology with $Q_{1}=0$ and $Q_{2}=0$ (Secs. $\mathrm{VA}$ and $\mathrm{VB}$ ) and then proceed to the case in which the couplings are present (Sec. VC).

\section{A. Scaling fixed points $B$ during the radiation and matter eras}

Let us first discuss the cosmology driven by the scaling solution B in the absence of the couplings $Q_{i}$. The scaling fixed point B corresponds to the one at which both the potential energies of the third and fourth terms in Eq. (5.1) provide non-vanishing contributions to $\Omega_{x}$, such that $y_{1} \neq 0$ and $y_{2} \neq 0$. From Eq. (5.9) there are two qualitatively different fixed points: (i) $\Omega_{r}=1-3 \Omega_{x} w_{x}$ and (ii) $\Omega_{r}=0$. In each case we obtain the following scaling fixed points $\mathrm{B} 1$ and $\mathrm{B} 2$ relevant to the cosmological dynamics during the radiation and matter eras, respectively. 


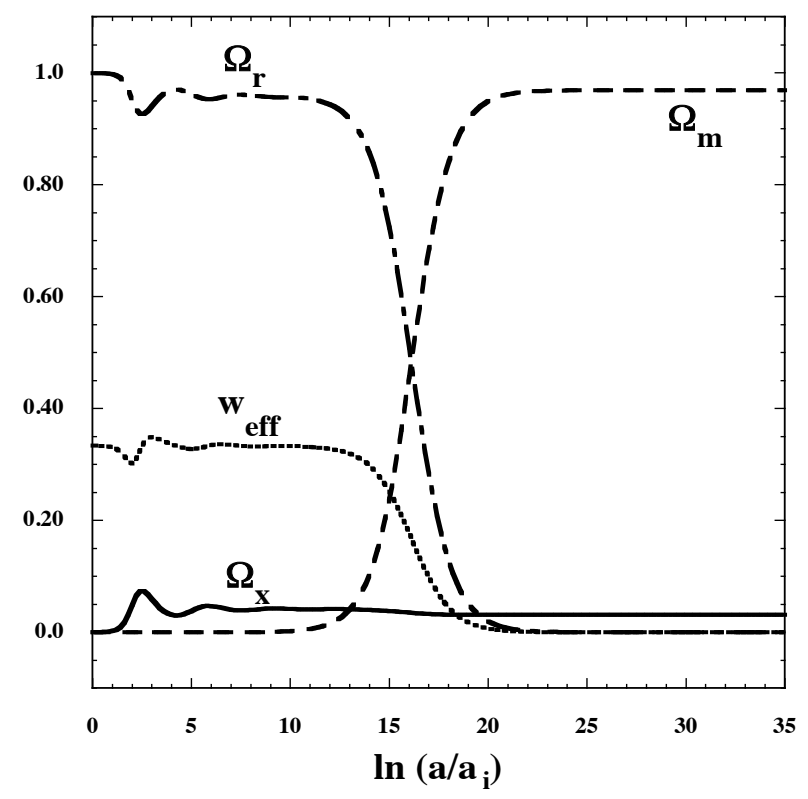

Figure 1: Evolution of $\Omega_{x}, \Omega_{r}, \Omega_{m}$, and $w_{\text {eff }}$ versus $\ln \left(a / a_{i}\right)$ (where $a_{i}$ is the initial value of $a$ ) for the model parameters $\lambda_{1}=13, \lambda_{2}=15, \mu_{1}=-3, \mu_{2}=-8, Q_{1}=0$, and $Q_{2}=0$. The initial conditions are chosen to be $x_{1}=0.01, x_{2}=0.02$, $y_{1}=0.005, y_{2}=0.005$, and $\Omega_{m}=1.0 \times 10^{-7}$.

- (i) Point B1

$$
x_{1}=\frac{2 \sqrt{6}}{3 \lambda_{1}}, \quad x_{2}=\frac{2 \sqrt{6}}{3 \lambda_{2}}, \quad y_{1}^{2}=\frac{4}{3\left(\mu_{2}-\mu_{1}\right) \lambda^{2}}\left(\mu_{2}+\frac{\lambda^{2}}{\lambda_{2}}\right), \quad y_{2}^{2}=\frac{4}{3\left(\mu_{1}-\mu_{2}\right) \lambda^{2}}\left(\mu_{1}+\frac{\lambda^{2}}{\lambda_{2}}\right),
$$

where $1 / \lambda^{2}=1 / \lambda_{1}^{2}+1 / \lambda_{2}^{2}$. At this point we have

$$
\Omega_{x}=\frac{4}{\lambda^{2}}, \quad \Omega_{r}=1-\frac{4}{\lambda^{2}}, \quad \Omega_{m}=0, \quad w_{\text {eff }}=w_{x}=\frac{1}{3} .
$$

- (ii) Point B2

$$
x_{1}=\frac{\sqrt{6}}{2 \lambda_{1}}, \quad x_{2}=\frac{\sqrt{6}}{2 \lambda_{2}}, \quad y_{1}^{2}=\frac{3}{2\left(\mu_{2}-\mu_{1}\right) \lambda^{2}}\left(\mu_{2}+\frac{\lambda^{2}}{\lambda_{2}}\right), \quad y_{2}^{2}=\frac{3}{2\left(\mu_{1}-\mu_{2}\right) \lambda^{2}}\left(\mu_{1}+\frac{\lambda^{2}}{\lambda_{2}}\right) .
$$

At this point we have

$$
\Omega_{x}=\frac{3}{\lambda^{2}}, \quad \Omega_{r}=0, \quad \Omega_{m}=1-\frac{3}{\lambda^{2}}, \quad w_{\mathrm{eff}}=w_{x}=0 .
$$

The existence of the above fixed points requires that $y_{1}^{2}>0$ and $y_{2}^{2}>0$, i.e.,

$$
\frac{1}{\mu_{2}-\mu_{1}}\left(\mu_{2}+\frac{\lambda^{2}}{\lambda_{2}}\right)>0 \quad \text { and } \quad \frac{1}{\mu_{1}-\mu_{2}}\left(\mu_{1}+\frac{\lambda^{2}}{\lambda_{2}}\right)>0 .
$$

Hence the signs of the terms $\mu_{2}+\lambda^{2} / \lambda_{2}$ and $\mu_{1}+\lambda^{2} / \lambda_{2}$ need to be opposite.

Perturbing Eqs. (5.5)-(5.9) about the point B1, we find that one of the eigenvalues is 1 and hence it is not stable. For the matter scaling point B2 we obtain the following five eigenvalues

$$
\begin{aligned}
& \gamma_{1}=-1, \quad \gamma_{2,3}=-\frac{3}{4}\left[1 \pm \sqrt{\frac{24}{\lambda^{2}}-7}\right], \\
& \gamma_{4,5}=-\frac{3}{4}\left[1 \pm \sqrt{1+\frac{8}{\lambda_{1}^{2} \lambda_{2}^{4}}\left[\mu_{1}\left(\lambda_{1}^{2}+\lambda_{2}^{2}\right)+\lambda_{1}^{2} \lambda_{2}\right]\left[\mu_{2}\left(\lambda_{1}^{2}+\lambda_{2}^{2}\right)+\lambda_{1}^{2} \lambda_{2}\right]}\right] .
\end{aligned}
$$




\begin{tabular}{|c|c|c|c|c|c|c|c|}
\hline & $x_{1}$ & $x_{2}$ & $y_{1}^{2}$ & $y_{2}^{2}$ & $\Omega_{x}$ & $\Omega_{r}$ & $w_{\text {eff }}$ \\
\hline \hline A & $\frac{\lambda^{2}}{\sqrt{6} \lambda_{1}}$ & $\frac{\lambda^{2}}{\sqrt{6} \lambda_{2}}$ & $\frac{\left[\lambda_{1}^{2}\left(\lambda_{2}^{2}-6\right)-6 \lambda_{2}^{2}\right]\left[\lambda_{1}^{2}\left(\lambda_{2}+\mu_{2}\right)+\lambda_{2}^{2} \mu_{2}\right]}{6\left(\mu_{1}-\mu_{2}\right)\left(\lambda_{1}^{2}+\lambda_{2}^{2}\right)^{2}}$ & $\frac{\left[\lambda_{1}^{2}\left(\lambda_{2}^{2}-6\right)-6 \lambda_{2}^{2}\right]\left[\lambda_{1}^{2}\left(\lambda_{2}+\mu_{1}\right)+\lambda_{2}^{2} \mu_{1}\right]}{6\left(\mu_{2}-\mu_{1}\right)\left(\lambda_{1}^{2}+\lambda_{2}^{2}\right)^{2}}$ & 1 & 0 & $-1+\frac{\lambda^{2}}{3}$ \\
\hline B1 & $\frac{2 \sqrt{6}}{3 \lambda_{1}}$ & $\frac{2 \sqrt{6}}{3 \lambda_{2}}$ & $\frac{4}{3\left(\mu_{2}-\mu_{1}\right) \lambda^{2}}\left(\mu_{2}+\frac{\lambda^{2}}{\lambda_{2}}\right)$ & $\frac{4}{3\left(\mu_{1}-\mu_{2}\right) \lambda^{2}}\left(\mu_{1}+\frac{\lambda^{2}}{\lambda_{2}}\right)$ & $\frac{4}{\lambda^{2}}$ & $1-\frac{4}{\lambda^{2}}$ & $\frac{1}{3}$ \\
\hline B2 & $\frac{\sqrt{6}}{2 \lambda_{1}}$ & $\frac{\sqrt{6}}{2 \lambda_{2}}$ & $\frac{3}{2\left(\mu_{2}-\mu_{1}\right) \lambda^{2}}\left(\mu_{2}+\frac{\lambda^{2}}{\lambda_{2}}\right)$ & $\frac{3}{2\left(\mu_{1}-\mu_{2}\right) \lambda^{2}}\left(\mu_{1}+\frac{\lambda^{2}}{\lambda_{2}}\right)$ & $\frac{3}{\lambda^{2}}$ & 0 & 0 \\
\hline C & $-\frac{\sqrt{6} Q_{1}}{3}$ & $-\frac{\sqrt{6} Q_{2}}{3}$ & 0 & 0 & $\frac{2}{3}\left(Q_{1}^{2}+Q_{2}^{2}\right)$ & 0 & $\frac{2}{3}\left(Q_{1}^{2}+Q_{2}^{2}\right)$ \\
\hline D & $x_{1}$ & $\pm \sqrt{1-x_{1}^{2}}$ & 0 & 0 & $\frac{4 \lambda_{2}^{2}}{\xi}$ & $1-\frac{4 \lambda_{2}^{2}}{\xi}$ & $\frac{1}{3}$ \\
\hline E1 & $\frac{2 \sqrt{6} \lambda_{1} \lambda_{2}\left(\lambda_{2}+\mu_{1}\right)}{3 \xi}$ & $-\frac{2 \sqrt{6} \lambda_{2}^{2} \mu_{1}}{3 \xi}$ & $\frac{4 \lambda_{2}^{2}}{3 \xi}$ & 0 & $\frac{3 \lambda_{2}^{2}}{\xi}$ & 0 & 0 \\
\hline E2 & $\frac{\sqrt{6} \lambda_{1} \lambda_{2}\left(\lambda_{2}+\mu_{1}\right)}{2 \xi}$ & $-\frac{\sqrt{6} \lambda_{2}^{2} \mu_{1}}{2 \xi}$ & $\frac{3 \lambda_{2}^{2}}{2 \xi}$ & 0 & 1 & 0 & $-1+\frac{\xi}{3 \lambda_{2}^{2}}$ \\
\hline F & $\frac{\sqrt{6} \lambda_{1}\left(\lambda_{2}+\mu_{1}\right)}{6 \lambda_{2}}$ & $-\frac{\sqrt{6} \mu_{1}}{6}$ & $1-\frac{\xi}{6 \lambda_{2}^{2}}$ & 0 & 0 & 0 \\
\hline
\end{tabular}

Table II: The properties of eight fixed points A, B1, B2, C, D, E1, E2, and F appearing in the model (5.1) in the presence of non-relativistic matter $\left(w_{m}=0\right)$ and radiation $\left(w_{m}=1 / 3\right)$. The quantity $\xi$ is defined by $\xi=\lambda_{2}^{2} \mu_{1}^{2}+\lambda_{1}^{2}\left(\lambda_{2}+\mu_{1}\right)^{2}$.

Provided $\lambda^{2}>3$, the eigenvalues $\gamma_{2,3}$ are either negative or complex with negative real parts. The condition $\lambda^{2}>3$ is equivalent to (4.29) with $w_{m}=0$. The eigenvalues $\gamma_{4,5}$ do not have positive real values for

$$
\left(\mu_{1}+\mu_{c}\right)\left(\mu_{2}+\mu_{c}\right)<0, \quad \text { where } \quad \mu_{c} \equiv \frac{\lambda_{1}^{2} \lambda_{2}}{\lambda_{1}^{2}+\lambda_{2}^{2}}=\frac{\lambda^{2}}{\lambda_{2}},
$$

which coincides with (4.22). As long as the conditions (5.16) hold, the condition (5.18) is satisfied.

For the existence of the scaling point B1 during the radiation era, we require that $\Omega_{x}<1$ and hence $\lambda^{2}>4$. Since the field density parameter is constrained to be $\Omega_{x}<0.045$ from the BBN [9], this puts the bound $\lambda>9.4$. In this case the fixed point B2 is stable under the condition (5.18). When the conditions $\lambda^{2}>4$ and (5.18) are satisfied, the eigenvalues $\gamma_{2,3}$ and $\gamma_{4,5}$ corresponding to the radiation fixed point B1 are negative (or complex with negative real parts) with $\gamma_{1}=1$, so it is a saddle point followed by the scaling matter solution B2.

In Fig. 1 we plot the evolution of the density parameters as well as the effective equation of state for $\lambda_{1}=13$, $\lambda_{2}=15, \mu_{1}=-3, \mu_{2}=-8, Q_{1}=0$, and $Q_{2}=0$. Since $\lambda=9.82$ and $\mu_{c}=6.43$ in this case, the stability condition (5.18) is satisfied. The initial conditions are chosen such that $y_{1}$ and $y_{2}$ as well as $x_{1}$ and $x_{2}$ are the similar orders to each other. As we see in Fig. 1, the solution temporally approaches the scaling radiation point B1 with $\Omega_{x}=4 / \lambda^{2}=4.14 \times 10^{-2}$ and $w_{\text {eff }}=1 / 3$. This is followed by the stable scaling matter point B2 characterized by $\Omega_{x}=3 / \lambda^{2}=3.11 \times 10^{-2}$ and $w_{\text {eff }}=0$. During the matter era, at least one of the eigenvalues for the $5 \times 5$ matrix associated with the perturbations about the fixed points A, C, and D shown in Table П are positive for the model parameters used in Fig. 1, so they are unstable. If $\mu_{1}$ and $\mu_{2}$ are outside the range (5.18), we also numerically confirmed that the fixed point B2 is not stable.

The above results show that, for the parameters $\lambda_{1} \gtrsim 10, \lambda_{2} \gtrsim 10$, and $\mu_{1}, \mu_{2}$ satisfying the condition (5.18), the solutions enter the scaling regime in which the field energy densities track the background energy density. This is a nice feature because even with initially large scalar-field energy densities the solutions approach the scaling matter fixed point B2 with $\Omega_{x} / \Omega_{m}=$ constant $<O(1)$. However, if we try to explain the late-time acceleration of the Universe as well, we need to consider different parameter spaces and initial conditions as compared to those given above. In Sec. $\mathrm{VB}$ we explore such a possibility for the model (5.1).

\section{B. Scaling solutions followed by cosmic acceleration}

If either $\lambda_{1}$ or $\lambda_{2}$ is smaller than the order of 1 , it is possible for the fixed point $\mathrm{A}$ to give rise to the late-time cosmic acceleration. In this section we study the case in which $\lambda_{1} \gtrsim O(10)$ and $\lambda_{2} \lesssim O(1)$ with $Q_{1}=0$ and $Q_{2}=0$.

The dark energy dynamics based on the separate exponential potentials characterized by the Lagrangian $P=$ $X_{1}+X_{2}-V_{1} e^{-\lambda_{1} \phi_{1}}-V_{2} e^{-\lambda_{2} \phi_{2}}$ have been studied in detail in Refs. 23 27. In such models, i.e., $\mu_{1}=0$ and $\mu_{2}=-\lambda_{2}$, the potential $V_{2} e^{-\lambda_{2} \phi_{2}}$ is suppressed relative to the other one $V_{1} e^{-\lambda_{1} \phi_{1}}$ during most of the cosmic expansion history, but the former comes out at late times to drive the accelerated expansion. In other words the quantities $y_{2}$ and $x_{2}$ are much smaller than 1 for the redshift $z=1 / a-1 \gg 1$, but they grow to the order of 1 around today $(a=1)$. If $\mu_{1}=0$, then it is clear from Eqs. (5.6) and (5.8) that the variables $x_{2}$ and $y_{2}$ remain to be much smaller than 1 for $z \gg 1$ because the field $\phi_{2}$ does not have a direct coupling to $\phi_{1}$.

When $\mu_{1} \neq 0$, however, the presence of the term $-\sqrt{6} \mu_{1} y_{1}^{2} / 2$ in Eq. (5.6) generally gives rise to a non-negligible kinetic energy of $\phi_{2}$ relative to $\phi_{1}$. As for the potential energy, Eq. (5.8) shows that $y_{2}$ can be much smaller than 
$y_{1}$ during most of the radiation and matter eras for $V_{2} \ll V_{1}$. Compared to the scaling fixed points B1 and B2, we search for other types of fixed points E1 (radiation era) and E2 (matter era) characterized by $y_{2}=0$ and non-zero values of $x_{1}, x_{2}$, and $y_{1}$. They are given, respectively, by

- (i) Point E1

$$
x_{1}=\frac{2 \sqrt{6} \lambda_{1} \lambda_{2}\left(\lambda_{2}+\mu_{1}\right)}{3 \xi}, \quad x_{2}=-\frac{2 \sqrt{6} \lambda_{2}^{2} \mu_{1}}{3 \xi}, \quad y_{1}^{2}=\frac{4 \lambda_{2}^{2}}{3 \xi}, \quad y_{2}^{2}=0
$$

where

$$
\xi \equiv \lambda_{2}^{2} \mu_{1}^{2}+\lambda_{1}^{2}\left(\lambda_{2}+\mu_{1}\right)^{2}
$$

At this point we have

$$
\Omega_{x}=\frac{4 \lambda_{2}^{2}}{\xi}, \quad \Omega_{r}=1-\frac{4 \lambda_{2}^{2}}{\xi}, \quad \Omega_{m}=0, \quad w_{\mathrm{eff}}=w_{x}=\frac{1}{3} .
$$

- (ii) Point E2

$$
x_{1}=\frac{\sqrt{6} \lambda_{1} \lambda_{2}\left(\lambda_{2}+\mu_{1}\right)}{2 \xi}, \quad x_{2}=-\frac{\sqrt{6} \lambda_{2}^{2} \mu_{1}}{2 \xi}, \quad y_{1}^{2}=\frac{3 \lambda_{2}^{2}}{2 \xi}, \quad y_{2}^{2}=0 .
$$

At this point we have

$$
\Omega_{x}=\frac{3 \lambda_{2}^{2}}{\xi}, \quad \Omega_{r}=0, \quad \Omega_{m}=1-\frac{3 \lambda_{2}^{2}}{\xi}, \quad w_{\mathrm{eff}}=w_{x}=0
$$

See Table If for the summary of fixed points relevant to the cosmological dynamics discussed in this section. Note that the condition $y_{1}^{2}>0$ is automatically satisfied for the points E1 and E2. In the $\mu_{1} \rightarrow 0$ limit we have $x_{2} \rightarrow 0$, so the field $\phi_{2}$ is effectively decoupled from the cosmological dynamics during the radiation and matter eras. If $\mu_{1} \neq 0$, then the kinetic energy of $\phi_{2}$ contributes to the dynamics such that the field density parameter $\Omega_{x}$ is modified relative to the case $\mu_{1}=0$. If the solution is in the scaling regime during the BBN epoch, the bound $\Omega_{x}<0.045$ translates to

$$
\frac{4 \lambda_{2}^{2}}{\lambda_{2}^{2} \mu_{1}^{2}+\lambda_{1}^{2}\left(\lambda_{2}+\mu_{1}\right)^{2}}<0.045
$$

The presence of the $\mu_{1}$ term allows the possibility of reducing the lhs of Eq. (5.24) relative to the case $\mu_{1}=0$.

Perturbing Eqs. (5.5)-(5.9) about the matter scaling solution E2, the eigenvalues of the matrix associated with the perturbations are given by

$$
\gamma_{1}=-\frac{3}{2}, \quad \gamma_{2}=-1, \quad \gamma_{3}=\frac{3}{2 \xi}\left[\left(\lambda_{1}^{2}+\lambda_{2}^{2}\right) \mu_{1}+\lambda_{1}^{2} \lambda_{2}\right]\left(\mu_{1}-\mu_{2}\right), \quad \gamma_{4,5}=-\frac{3}{4}\left[1 \pm \sqrt{\frac{24 \lambda_{2}^{2}}{\xi}-7}\right]
$$

The point E2 is stable under the conditions

$$
\begin{aligned}
& {\left[\left(\lambda_{1}^{2}+\lambda_{2}^{2}\right) \mu_{1}+\lambda_{1}^{2} \lambda_{2}\right]\left(\mu_{1}-\mu_{2}\right)<0,} \\
& \xi>3 \lambda_{2}^{2} .
\end{aligned}
$$

If $\mu_{1}=0$ and $\mu_{2}=-\lambda_{2}$, for example, the condition (5.26) is violated (whereas the condition (5.27) translates to $\left.\lambda_{1}^{2}>3\right)$, so that the matter point E2 is a saddle. In order to realize the late-time accelerated expansion, either (5.26) or (5.27) should be at least violated to exit from the scaling matter era. Two of the eigenvalues for the perturbations about the radiation point E1 are 1 and -1 , so it is a saddle point.

The matter scaling point E2 can be followed by the scalar-field dominated solution A discussed in Sec. III. For the model (5.1) the fixed point A corresponds to $x_{1}=\lambda^{2} /\left(\sqrt{6} \lambda_{1}\right), x_{2}=\lambda^{2} /\left(\sqrt{6} \lambda_{2}\right)$, and

$$
y_{1}^{2}=\frac{\left[\lambda_{1}^{2}\left(\lambda_{2}^{2}-6\right)-6 \lambda_{2}^{2}\right]\left[\lambda_{1}^{2}\left(\lambda_{2}+\mu_{2}\right)+\lambda_{2}^{2} \mu_{2}\right]}{6\left(\mu_{1}-\mu_{2}\right)\left(\lambda_{1}^{2}+\lambda_{2}^{2}\right)^{2}}, \quad y_{2}^{2}=\frac{\left[\lambda_{1}^{2}\left(\lambda_{2}^{2}-6\right)-6 \lambda_{2}^{2}\right]\left[\lambda_{1}^{2}\left(\lambda_{2}+\mu_{1}\right)+\lambda_{2}^{2} \mu_{1}\right]}{6\left(\mu_{2}-\mu_{1}\right)\left(\lambda_{1}^{2}+\lambda_{2}^{2}\right)^{2}} .
$$



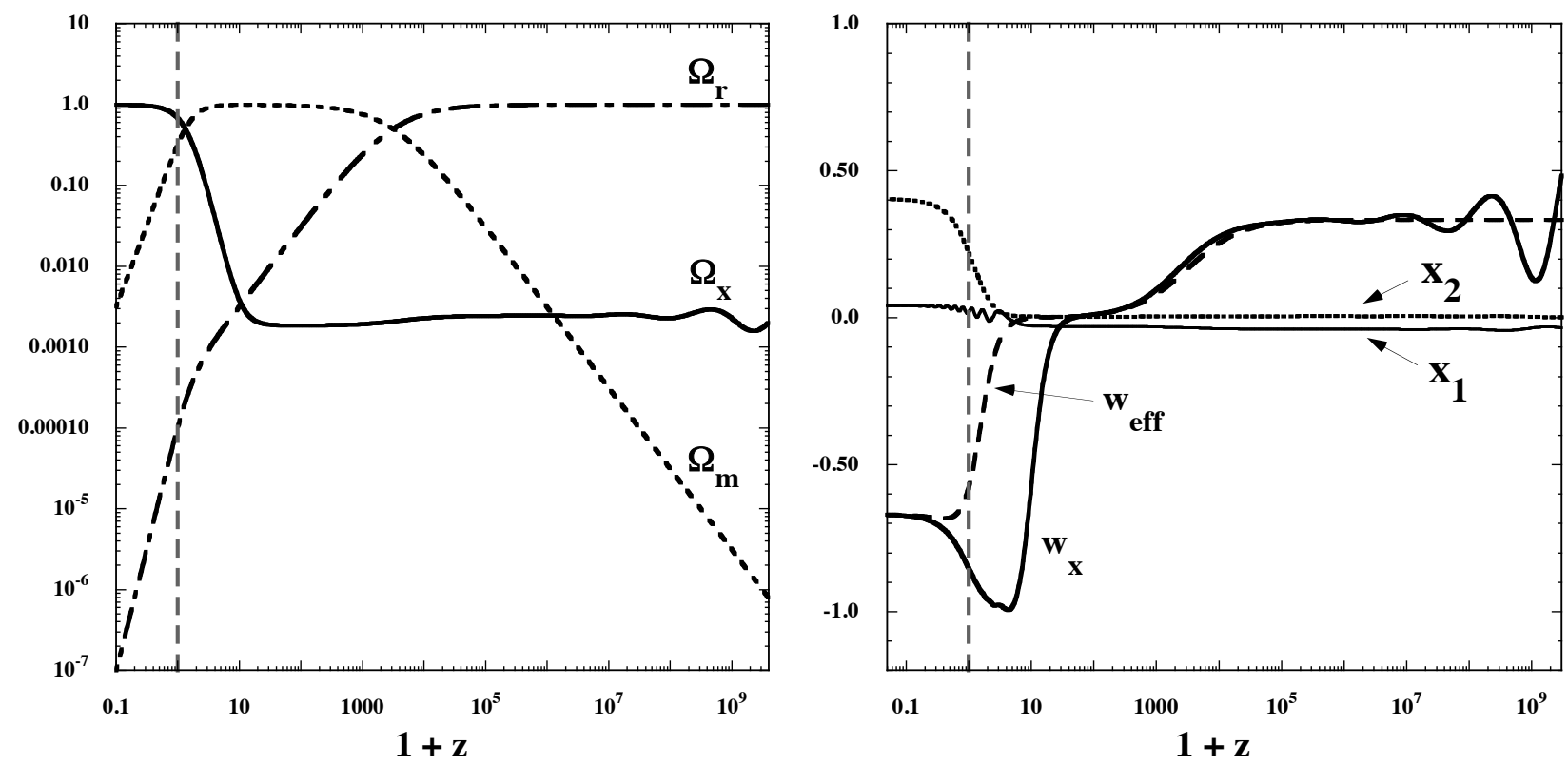

Figure 2: (Left) Evolution of $\Omega_{x}, \Omega_{r}$ and $\Omega_{m}$ versus $1+z$ for the model parameters $\lambda_{1}=10, \lambda_{2}=1, \mu_{1}=-5, \mu_{2}=-1 / 2$, $Q_{1}=0$, and $Q_{2}=0$. The initial conditions are chosen to be $x_{1}=-0.04, x_{2}=0, y_{1}=0.02, y_{2}=6.3 \times 10^{-20}$, and $\Omega_{m}=8.0 \times 10^{-7}$. (Right) Evolution of $w_{\text {eff }}, w_{x}, x_{1}$, and $x_{2}$ for the same model parameters and the initial conditions as those in the left panel. The vertical dashed line corresponds to the present epoch $(z=0)$.

The eigenvalues of the matrix for the perturbations about the point A are given by

$$
\begin{aligned}
& \gamma_{1}=\lambda^{2}-4, \quad \gamma_{2}=\lambda^{2}-3, \quad \gamma_{3}=\frac{1}{2}\left(\lambda^{2}-6\right), \\
& \gamma_{4,5}=\frac{\lambda^{2}-6}{4}\left[1 \pm \sqrt{1+\frac{8 \lambda^{2}\left[\left(\lambda_{1}^{2}+\lambda_{2}^{2}\right) \mu_{1}+\lambda_{1}^{2} \lambda_{2}\right]\left[\left(\lambda_{1}^{2}+\lambda_{2}^{2}\right) \mu_{2}+\lambda_{1}^{2} \lambda_{2}\right]}{\lambda_{1}^{2} \lambda_{2}^{4}\left(6-\lambda^{2}\right)}}\right] .
\end{aligned}
$$

If we impose the condition

$$
\lambda^{2}<2,
$$

for the realization of cosmic acceleration, we find that the fixed point $\mathrm{A}$ is stable under the condition

$$
\left(\mu_{1}+\mu_{c}\right)\left(\mu_{2}+\mu_{c}\right)<0,
$$

which is the same as (5.18). The above results are consistent with the general stability analysis performed in Sec. IV]

As long as the condition (5.31) holds, the condition (5.26) is violated. Hence the matter scaling point E2 is in fact a saddle that can be followed by the attractor solution A. When $\lambda$ is in the range (5.30), the eigenvalue $\gamma_{3}$ for the matter scaling fixed point B2 in Eq. (5.17) is positive. In this case the scaling matter fixed point B2 cannot be a stable attractor.

In Fig. 22 we plot the evolution of the density parameters as well as $w_{\text {eff }}, w_{x}, x_{1}, x_{2}$ for $\lambda_{1}=10, \lambda_{2}=1, \mu_{1}=-5$, $\mu_{2}=-1 / 2$ with the initial conditions $x_{1}=-0.04, x_{2}=0, y_{1}=0.02, y_{2}=6.3 \times 10^{-20}$, and $\Omega_{m}=8.0 \times 10^{-7}$. In this case the condition (5.26) does not hold, whereas both the conditions (5.30) and (5.31) are satisfied. Hence the saddle matter point E2 is followed by the stable attractor A with cosmic acceleration.

The field density parameter computed from Eqs. (5.21) and (5.23) are given by $\Omega_{x}=2.46 \times 10^{-3}$ and $\Omega_{x}=$ $1.85 \times 10^{-3}$ during the radiation and matter eras, respectively. These values show good agreement with the numerical simulation of Fig. 2. We also confirmed that the variables $x_{1}, x_{2}$, and $y_{1}^{2}$ first approach the radiation scaling solution E1 given by Eq. (5.19) and then they evolve to the matter scaling solution E2 characterized by Eq. (5.22). From Eq. (5.22) we have $x_{1}=-3.01 \times 10^{-2}$ and $x_{2}=3.77 \times 10^{-3}$ during the matter-dominated epoch (see the right panel of Fig. (2), so the field kinetic energy of $\phi_{2}$ is non-negligible relative to that of $\phi_{1}$.

The variable $y_{2}$ is much smaller than 1 for the redshift $z \gg 1$, but it grows to the order of 1 for $z<O(1)$. For the model parameters used in Fig. 2, we have $\lambda=0.995, x_{1}=4.04 \times 10^{-2}, x_{2}=0.404, y_{1}^{2}=9.09 \times 10^{-2}$, and 

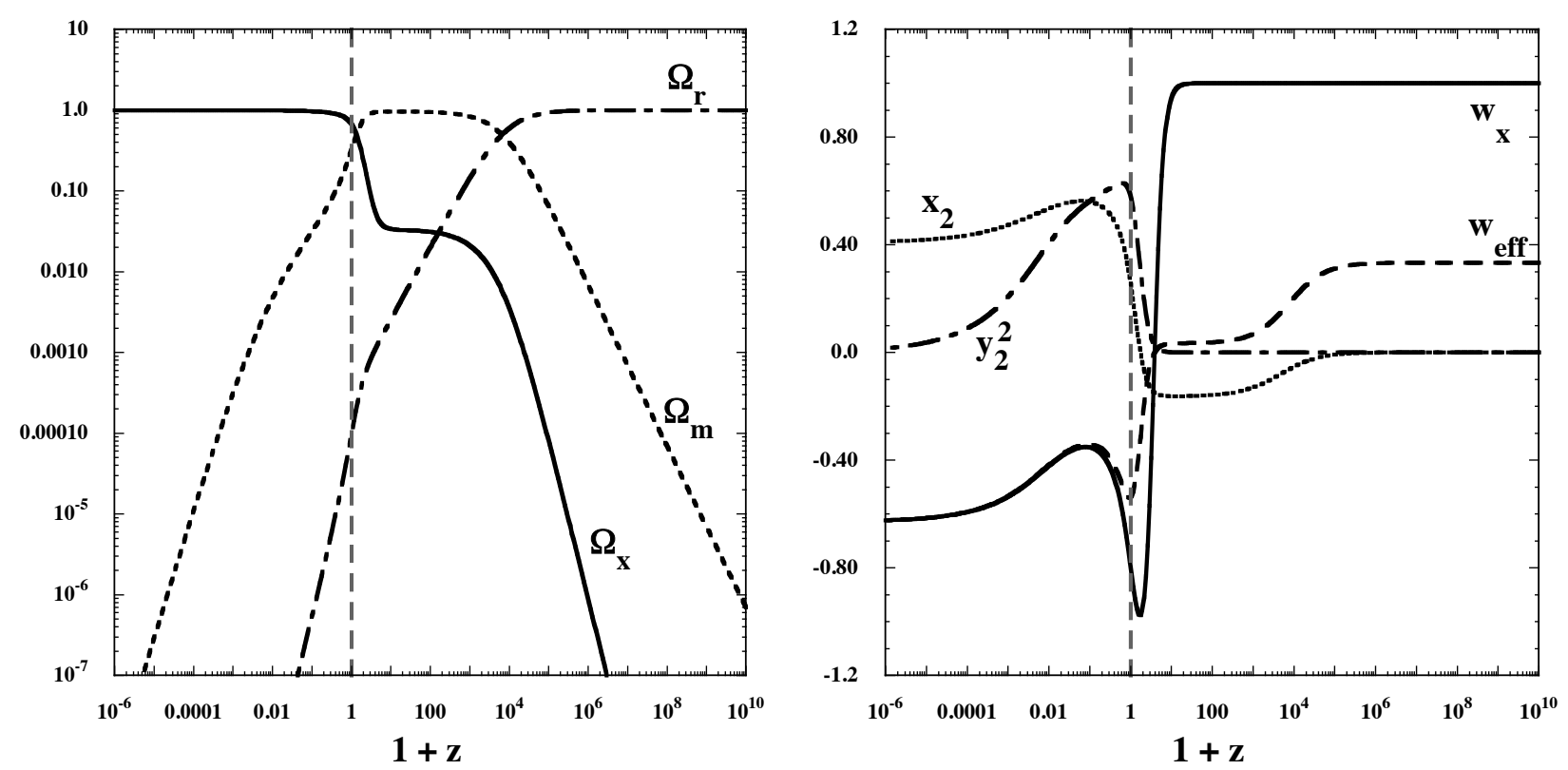

Figure 3: (Left) Evolution of $\Omega_{x}, \Omega_{r}$ and $\Omega_{m}$ versus $1+z$ for the model parameters $\lambda_{1}=1, \lambda_{2}=1.5, \mu_{1}=-1, \mu_{2}=-1.5$, $Q_{1}=0.1$, and $Q_{2}=0.2$. The initial conditions are chosen to be $x_{1}=-2.0 \times 10^{-8}, x_{2}=-3.0 \times 10^{-8}, y_{1}^{2}=1.0 \times 10^{-40}$, $y_{2}^{2}=1.0 \times 10^{-39}$, and $\Omega_{m}=4.0 \times 10^{-7}$. (Right) Evolution of $w_{\text {eff }}, w_{x}, x_{2}$, and $y_{2}^{2}$ for the same model parameters and the initial conditions as those in the left panel. The vertical dashed line corresponds to the present epoch $(z=0)$.

$y_{2}^{2}=0.744$ at the fixed point A. As we see in the right panel of Fig. 2. the solutions finally approach the fixed point A characterized by $w_{\text {eff }}=w_{x}=-1+\lambda^{2} / 3$. In the early radiation era the dark energy equation of state $w_{x}$ exhibits an oscillation before reaching the radiation scaling point $\mathrm{E} 1\left(w_{x}=1 / 3\right)$ and then it evolves to 0 during the matter era. The decrease of $w_{x}$ from 0 occurs around $z=O(10)$ and then $w_{x}$ reaches a minimum around -1 for $z \gtrsim O(1)$. Finally, $w_{x}$ approaches the asymptotic value $-1+\lambda^{2} / 3$ with $\Omega_{x}=1$.

The fixed point A corresponds to the assisted inflationary attractor with $\lambda$ satisfying $1 / \lambda^{2}=1 / \lambda_{1}^{2}+1 / \lambda_{2}^{2}$. Even if each $\lambda_{i}^{2}$ is larger than 2 , it is possible to realize $\lambda^{2}<2$ and $w_{\text {eff }}=w_{x}<-1 / 3$. The presence of more than two scalar fields allows the possibility of reducing $\lambda$ further.

\section{C. $\phi \mathrm{MDE}$ followed by cosmic acceleration}

In the presence of the couplings $Q_{1}$ and $Q_{2}$, the standard matter era can be replaced by the $\phi \mathrm{MDE}$. The latter corresponds to the case with a negligible potential energy relative to kinetic energies of scalar fields, such that $y_{1}=0$ and $y_{2}=0$. The $\phi \mathrm{MDE}$ is characterized by the fixed point

$$
x_{1}=-\frac{\sqrt{6} Q_{1}}{3}, \quad x_{2}=-\frac{\sqrt{6} Q_{2}}{3}, \quad y_{1}=0, \quad y_{2}=0, \quad \Omega_{r}=0 .
$$

The eigenvalues of the matrix for perturbations about the point (5.32) are given by

$$
\begin{aligned}
& \gamma_{1}=2\left(Q_{1}^{2}+Q_{2}^{2}\right)-1, \quad \gamma_{2,3}=Q_{1}^{2}+Q_{2}^{2}-\frac{3}{2}, \quad \gamma_{4}=\frac{3}{2}+Q_{1}^{2}+Q_{2}\left(Q_{2}-\mu_{1}\right)+\frac{\lambda_{1}}{\lambda_{2}}\left(\mu_{1}+\lambda_{2}\right) Q_{1}, \\
& \gamma_{5}=\frac{3}{2}+Q_{1}^{2}+Q_{2}\left(Q_{2}-\mu_{2}\right)+\frac{\lambda_{1}}{\lambda_{2}}\left(\mu_{2}+\lambda_{2}\right) Q_{1} .
\end{aligned}
$$

In the limit $Q_{1} \rightarrow 0$ and $Q_{2} \rightarrow 0$ we have $\gamma_{1} \rightarrow-1, \gamma_{2,3} \rightarrow-3 / 2$, and $\gamma_{4,5} \rightarrow 3 / 2$, so the point (5.32) is a saddle.

In the following we shall study the case $\lambda_{1} \lesssim O(1)$ and $\lambda_{2} \lesssim O(1)$ with the initial values of $\left|y_{1}\right|$ and $\left|y_{2}\right|$ much smaller than 1. In this case the potential energy of the scalar fields is much smaller than the energy density of the background fluid by the end of the matter era. If we choose the initial conditions where either $y_{1}$ or $y_{2}$ is not very close to 0, the solutions can approach other fixed points like E1 and E2 discussed in Sec. VB. 
In Fig. 3 we plot the evolution of the density parameters as well as $w_{\text {eff }}, w_{x}, x_{2}$, and $y_{2}^{2}$ for $\lambda_{1}=1, \lambda_{2}=1.5$, $\mu_{1}=-1, \mu_{2}=-1.5, Q_{1}=0.1$, and $Q_{2}=0.2$ with the initial values of $y_{1}$ and $y_{2}$ close to 0 . During the radiation era the field density parameter $\Omega_{x}$ is much smaller than $\Omega_{r}$, but it temporarily approaches the $\phi$ MDE characterized by

$$
\Omega_{x}=w_{\text {eff }}=\frac{2}{3}\left(Q_{1}^{2}+Q_{2}^{2}\right), \quad w_{x}=1 .
$$

These analytic values show good agreement with the numerical simulation of Fig. 3 (i.e., $\Omega_{x}=w_{\text {eff }} \simeq 0.033$ with $\left.x_{2} \simeq-0.163\right)$. The dark energy equation of state $w_{x}$ is close to 1 during the deep matter era. This property can be observationally distinguished from the case shown in Fig. 2 (i.e., $w_{x} \simeq 0$ ).

In Fig. 3 the solution exits from the $\phi \mathrm{MDE}$ to the epoch of cosmic acceleration. In fact, $w_{x}$ decreases to the minimum close to -1 by today and then it starts to grow to the asymptotic value around -0.63 . For the model parameters used in Fig. 3 the attractor is different from the fixed point A discussed in Sec. VB. In fact one of the eigenvalues in Eq. (5.29) is positive $\left(\gamma_{5}=0.649\right)$, so the point A is not stable. In this case the solutions approach another fixed point $\mathrm{F}$ characterized by

$$
x_{1}=\frac{\sqrt{6} \lambda_{1}\left(\lambda_{2}+\mu_{1}\right)}{6 \lambda_{2}}, \quad x_{2}=-\frac{\sqrt{6} \mu_{1}}{6}, \quad y_{1}^{2}=1-\frac{\xi}{6 \lambda_{2}^{2}}, \quad y_{2}^{2}=0
$$

and

$$
w_{\text {eff }}=w_{x}=-1+\frac{\xi}{3 \lambda_{2}^{2}}, \quad \Omega_{x}=1, \quad \Omega_{r}=0,
$$

where $\xi$ is defined by Eq. (5.20). The eigenvalues of the matrix for perturbations about the point $\mathrm{F}$ are

$$
\begin{aligned}
& \gamma_{1}=-4+\frac{\xi}{\lambda_{2}^{2}}, \quad \gamma_{2,3}=-3+\frac{\xi}{2 \lambda_{2}^{2}}, \quad \gamma_{4}=\frac{1}{2 \lambda_{2}^{2}}\left[\mu_{1}\left(\lambda_{1}^{2}+\lambda_{2}^{2}\right)+\lambda_{1}^{2} \lambda_{2}\right]\left(\mu_{1}-\mu_{2}\right), \\
& \gamma_{5}=-3+\mu_{1}^{2}+\lambda_{1}\left(Q_{1}+\lambda_{1}\right)-Q_{2} \mu_{1}+\frac{\lambda_{1}}{\lambda_{2}} \mu_{1}\left(Q_{1}+2 \lambda_{1}\right)+\mu_{1}^{2} \frac{\lambda_{1}^{2}}{\lambda_{2}^{2}} .
\end{aligned}
$$

The accelerated expansion can be realized for

$$
\xi<2 \lambda_{2}^{2}
$$

in which case $\gamma_{1}, \gamma_{2}, \gamma_{3}$ are negative. The eigenvalue $\gamma_{4}$ is negative for

$$
\left(\mu_{1}+\lambda^{2} / \lambda_{2}\right)\left(\mu_{1}-\mu_{2}\right)<0 .
$$

This inequality is opposite to that of the stability condition (4.21) derived for the fixed points A and B in the absence of the couplings $Q_{i}$. We also require $\gamma_{5}<0$ to ensure the stability of the point $\mathrm{F}$.

For the model parameters used in Fig. 3 all the eigenvalues (5.37) are negative, so the point F is in fact stable. Since $y_{2}=0$ at the point $\mathrm{F}$, the potential $V_{2} e^{-\lambda_{1}\left(1+\mu_{2} / \lambda_{2}\right) \phi_{1}+\mu_{2} \phi_{2}}$ does not contribute to the dynamics (see the evolution of $y_{2}^{2}$ in Fig. 31). The acceleration of the Universe is driven by the potential $V_{1} e^{-\lambda_{1}\left(1+\mu_{1} / \lambda_{2}\right) \phi_{1}+\mu_{1} \phi_{2}}$. Numerically we confirmed that the asymptotic values of $w_{x}, w_{\text {eff }}, x_{1}, x_{2}$, and $y_{1}^{2}$ show good agreement with the analytic estimation given above. Depending on the model parameters $\lambda_{1}, \lambda_{2}, \mu_{1}, \mu_{2}, Q_{1}$, and $Q_{2}$, the attractor solutions are different. Evaluating the eigenvalues (5.29) and (5.37) in each case, we readily know the stability of the fixed points A and F.

Finally we briefly discuss the case in which the point B may be responsible for the scaling attractor $\left(\Omega_{x} \simeq 0.7\right)$ with cosmic acceleration. As we mentioned above, if the scaling solution $\mathrm{B}$ is stable, the fixed point $\mathrm{F}$ is not stable. Setting $w_{m}=0$ in Eq. (3.32), the condition $\Omega_{x}<1$ translates to $q>3 / \lambda^{2}-1$. Moreover the accelerated expansion is realized for $w_{\text {eff }}=-q /(1+q)<-1 / 3$, that is, $q>1 / 2$. These two conditions can be written as

$$
\frac{Q_{1}}{\lambda_{1}}+\frac{Q_{2}}{\lambda_{2}}>3\left(\frac{1}{\lambda_{1}^{2}}+\frac{1}{\lambda_{2}^{2}}\right)-1, \quad \frac{Q_{1}}{\lambda_{1}}+\frac{Q_{2}}{\lambda_{2}}>\frac{1}{2} .
$$

On the other hand, the $\phi \mathrm{MDE}$ can be compatible with the CMB observations for $w_{\text {eff }}=\Omega_{x}=2\left(Q_{1}^{2}+Q_{2}^{2}\right) / 3 \ll 1$ [31], i.e.,

$$
Q_{1}^{2}+Q_{2}^{2} \ll 1
$$

For the existence of the scaling accelerated point B we require the large couplings $Q_{1}$ and $Q_{2}$ satisfying the conditions (5.40), but the presence of the acceptable $\phi \mathrm{MDE}$ demands the small couplings. In particular we need small values of $\lambda_{1}$ and $\lambda_{2}$ to make $Q_{1} / \lambda_{1}+Q_{2} / \lambda_{2}$ large, but in this case the rhs on the first inequality of Eq. (5.40) tends to be even larger. Hence, as in the single-field case [17], it is generally difficult to realize the sequence of the $\phi \mathrm{MDE}$ followed by the scaling solution $\mathrm{B}$ with $\Omega_{x} \simeq 0.7$ and $w_{\text {eff }}<-1 / 3$. 


\section{CONCLUSIONS}

In general k-essence model with multiple scalar fields $\phi_{i}(i=1, \cdots, N)$, we derived the Lagrangian for the existence of cosmological scaling solutions in the presence of a barotropic perfect fluid coupled to $\phi_{i}$. The resulting Lagrangian is simply given by Eq. (2.21), where $g$ is an arbitrary function in terms of $Y_{i}=X_{i} e^{\lambda_{1} \phi_{1}}$ and $Z_{i}=\phi_{i+1}-\lambda_{1} \phi_{1} / \lambda_{i+1}$. Along the scaling solution, the scalar fields evolve as Eq. (2.23) with $Y_{i}$ and $Z_{i}$ constant. For canonical multiple scalar fields the scaling solution behaves as an effective single field $\sigma$ with the trajectory given by Eq. (2.36).

For the multi-field Lagrangian (2.21) we obtained the autonomous equations (3.5)-(3.8) by introducing the dimensionless variables $x_{i}=\dot{\phi}_{i} /(\sqrt{6} H)$ and $y=e^{-\lambda_{1} \phi_{1} / 2} /(\sqrt{3} H)$. There are two important fixed points with $y \neq 0$ for arbitrary functions of $g$. One of them is the scalar-field dominated point $A\left(\Omega_{x}=1\right)$ satisfying the conditions (3.20)(3.25). We showed that the assisted inflationary mechanism is present for the fixed point A. Even if each field does not lead to cosmic acceleration, the multiple fields can cooperatively do so by reducing the effective slope $\lambda$ defined by Eq. (3.24). Provided $\lambda^{2}<2$, the point A can be responsible for the accelerated expansion. Another fixed point B is the scaling solution $\left(\Omega_{x}=\right.$ constant generally different from 1 ) satisfying the conditions (3.28)-(3.33). In the presence of the couplings between the fields and the background fluid $(q \neq 0)$, the effective equation of state $w_{\text {eff }}$ for the point $\mathrm{B}$ is generally different from $w_{m}$.

If the Lagrangian (2.21) is specified to some form, we can show the existence of kinetically driven fixed points satisfying $y=0$. For the function (3.37), which involves the case of $N$ canonical scalar fields, there exist the $\phi \mathrm{MDE}$ point $\mathrm{C}$ characterized by $x_{i}=\sqrt{6} Q_{i} /\left[3 c_{i}\left(w_{m}-1\right)\right]$ and the kinetic point $\mathrm{D}$ with $\Omega_{x}=1$. Unlike the fixed points $\mathrm{A}$ and $\mathrm{B}$, the quantities $y$ and $Z_{i}$ are not necessarily constant for $\mathrm{C}$ and $\mathrm{D}$. In the presence of the couplings $Q_{i}$, the standard matter-dominated epoch can be replaced by the $\phi \mathrm{MDE}$ with $w_{\mathrm{eff}}=\Omega_{x}=\sum_{i=1}^{N} 2 Q_{i}^{2} /\left(3 c_{i}\right)$.

In Sec. IV we studied the stability of the fixed points A and B in the two-field system described by the Lagrangian (4.1). For the point $\mathrm{A}$ it is possible to carry out the general analysis without specifying any functional form of $g$. The stability of this scalar-field dominated solution is ensured under the conditions (4.5), (4.12), and (4.13). The stability analysis for the point B is too cumbersome to be written in a general way. In the model of two canonical fields with the function (4.23), we showed that the scaling solution B is stable under the conditions (4.29) and (4.30) with the additional requirement (4.25). These conditions can be nicely interpreted as a geometric approach based on the rotation in the field space. In the case of two canonical fields with $Q_{i}=0$, we found that the point A is stable (unstable) when the point B is unstable (stable).

In Sec. $\mathrm{V}$ we discussed the cosmological dynamics for the two-field model (5.1) in the presence of radiation and non-relativistic matter. In doing so, it is convenient to employ the variables $y_{1}$ and $y_{2}$ defined in Eq. (5.2) instead of $y$ and $Z_{1}$. In this model there exist a few more fixed points than $\mathrm{A}, \mathrm{B}, \mathrm{C}, \mathrm{D}$ relevant to the scaling radiation/matter eras and the epoch of cosmic acceleration. These fixed points are summarized in Table II]

Depending on the model parameters and initial conditions of the model (5.1), there are several qualitatively different cases: (i) the scaling radiation era (B1) followed by the stable scaling matter era (B2), (ii) the scaling radiation (E1) and matter (E2) eras followed by the point A with cosmic acceleration, and (iii) the $\phi \mathrm{MDE}$ point $\mathrm{C}$ followed by the accelerated expansion driven by another point $\mathrm{F}$. The points E1, E2, and F, which satisfy $y_{2}=0$, arise when the potential energy of the last term of Eq. (5.1) is negligibly small relative to the third term. The cases (ii) and (iii) can be distinguished from each other by the different evolution of the dark energy equation of state $w_{x}$.

It will be of interest to put observational bounds on the viable parameter space of the model (5.1) and its extended model by using the data of supernovae type Ia, CMB, baryonic acoustic oscillations, and the BBN. The BBN bound should not be so restrictive in the multi-field context because the field density parameter $\Omega_{x}$ in the radiation era can be smaller than that in the single-field case. The study of matter density perturbations is also important to place constraints on the couplings $Q_{i}$ in the presence of the $\phi \mathrm{MDE}$. We leave these topics for a future work.

\section{ACKNOWLEDGEMENTS}

We thank Luca Amendola for useful discussions. This work is supported by the Grant-in-Aid for Scientific Research from JSPS (Nos. 24540287 (TC), 24540286 (ST)), by the Grant-in-Aid for Scientific Research on Innovative Areas (No. 21111006 (ST)) and in part by Nihon University (TC). Some of this work was done at the Yukawa Institute, during the workshop YITP-X-13-03.

[1] D. Kazanas, Astrophys. J. 241 L59 (1980); K. Sato, Mon. Not. R. Astron. Soc. 195, 467 (1981); Phys. Lett. 99B, 66 (1981); A. H. Guth, Phys. Rev. D 23, 347 (1981). 
[2] P. A. R. Ade et al. [Planck Collaboration], arXiv:1303.5076 [astro-ph.CO].

[3] Y. Fujii, Phys. Rev. D 26, 2580 (1982); L. H. Ford, Phys. Rev. D 35, 2339 (1987); C. Wetterich, Nucl. Phys B. 302, 668 (1988).

[4] T. Chiba, N. Sugiyama and T. Nakamura, Mon. Not. Roy. Astron. Soc. 289, L5 (1997) astro-ph/9704199; P. G. Ferreira and M. Joyce, Phys. Rev. Lett. 79, 4740 (1997) astro-ph/9707286; R. R. Caldwell, R. Dave and P. J. Steinhardt, Phys. Rev. Lett. 80, 1582 (1998) astro-ph/9708069]; T. Chiba, N. Sugiyama and T. Nakamura, Mon. Not. Roy. Astron. Soc. 301, 72 (1998) astro-ph/9806332; I. Zlatev, L. -M. Wang and P. J. Steinhardt, Phys. Rev. Lett. 82, 896 (1999) astro-ph/9807002.

[5] S. Weinberg, Rev. Mod. Phys. 61, 1 (1989); V. Sahni and A. A. Starobinsky, Int. J. Mod. Phys. D 9, 373 (2000) astro-ph/9904398; S. M. Carroll, Living Rev. Rel. 4, 1 (2001) astro-ph/0004075]; T. Padmanabhan, Phys. Rept. 380, 235 (2003) hep-th/0212290; P. J. E. Peebles and B. Ratra, Rev. Mod. Phys. 75, 559 (2003) astro-ph/0207347]; E. J. Copeland, M. Sami and S. Tsujikawa, Int. J. Mod. Phys. D 15, 1753 (2006) hep-th/0603057]; S. Tsujikawa, arXiv:1004.1493 [astroph.CO]; S. Tsujikawa, Class. Quant. Grav. 30, 214003 (2013) [arXiv:1304.1961 [gr-qc]].

[6] P. J. E. Peebles and B. Ratra, Astrophys. J. 325, L17 (1988); B. Ratra and P. J. E. Peebles, Phys. Rev. D 37,3406 (1988).

[7] E. J. Copeland, A. R. Liddle and D. Wands, Phys. Rev. D 57, 4686 (1998) gr-qc/9711068.

[8] P. G. Ferreira and M. Joyce, Phys. Rev. D 58, 023503 (1998) astro-ph/9711102.

[9] R. Bean, S. H. Hansen and A. Melchiorri, Phys. Rev. D 64, 103508 (2001) astro-ph/0104162.

[10] C. Armendariz-Picon, T. Damour and V. F. Mukhanov, Phys. Lett. B 458, 209 (1999) hep-th/9904075].

[11] T. Chiba, T. Okabe and M. Yamaguchi, Phys. Rev. D 62, 023511 (2000) astro-ph/9912463; C. Armendariz-Picon, V. F. Mukhanov and P. J. Steinhardt, Phys. Rev. Lett. 85, 4438 (2000) astro-ph/0004134; T. Chiba, Phys. Rev. D 66, 063514 (2002) astro-ph/0206298.

[12] F. Piazza and S. Tsujikawa, JCAP 0407, 004 (2004) hep-th/0405054.

[13] S. Tsujikawa and M. Sami, Phys. Lett. B 603, 113 (2004).

[14] S. Tsujikawa and M. Sami, JCAP 0701, 006 (2007) hep-th/0608178.

[15] A. R. Gomes and L. Amendola, arXiv:1306.3593 [astro-ph.CO].

[16] S. Tsujikawa, Phys. Rev. D 73, 103504 (2006) hep-th/0601178.

[17] L. Amendola, M. Quartin, S. Tsujikawa and I. Waga, Phys. Rev. D 74, 023525 (2006) astro-ph/0605488.

[18] T. Barreiro, E. J. Copeland and N. J. Nunes, Phys. Rev. D 61, 127301 (2000) astro-ph/9910214.

[19] V. Sahni and L. -M. Wang, Phys. Rev. D 62, 103517 (2000) astro-ph/9910097; A. Albrecht and C. Skordis, Phys. Rev. Lett. 84, 2076 (2000) astro-ph/9908085; S. Dodelson, M. Kaplinghat and E. Stewart, Phys. Rev. Lett. 85, 5276 (2000) astro-ph/0002360; L. A. Urena-Lopez and T. Matos, Phys. Rev. D 62, 081302 (2000) astro-ph/0003364.

[20] T. Chiba, A. De Felice and S. Tsujikawa, Phys. Rev. D 87, 083505 (2013) arXiv:1210.3859 [astro-ph.CO]].

[21] A. R. Liddle, A. Mazumdar and F. E. Schunck, Phys. Rev. D 58, 061301 (1998) astro-ph/9804177].

[22] K. A. Malik and D. Wands, Phys. Rev. D 59, 123501 (1999) astro-ph/9812204; E. J. Copeland, A. Mazumdar and N. J. Nunes, Phys. Rev. D 60, 083506 (1999) astro-ph/9904309.

[23] Z. K. Guo, Y. -S. Piao and Y. -Z. Zhang, Phys. Lett. B 568, 1 (2003) hep-th/0304048.

[24] D. Blais and D. Polarski, Phys. Rev. D 70, 084008 (2004) astro-ph/0404043.

[25] S. A. Kim, A. R. Liddle and S. Tsujikawa, Phys. Rev. D 72, 043506 (2005) astro-ph/0506076].

[26] J. Ohashi and S. Tsujikawa, Phys. Rev. D 80, 103513 (2009) [arXiv:0909.3924 [gr-qc]].

[27] C. van de Bruck and J. M. Weller, Phys. Rev. D 80, 123014 (2009) [arXiv:0910.1934 [astro-ph.CO]].

[28] Z. -K. Guo, Y. -S. Piao, R. -G. Cai and Y. -Z. Zhang, Phys. Lett. B 576, 12 (2003) hep-th/0306245.

[29] A. J. Tolley and D. H. Wesley, JCAP 0705, 006 (2007) hep-th/0703101.

[30] M. Dodelson, X. Dong, E. Silverstein and G. Torroba, arXiv:1310.5297 [hep-th].

[31] L. Amendola, Phys. Rev. D 62, 043511 (2000) astro-ph/9908023.

[32] C. Brans and R. H. Dicke, Phys. Rev. 124, 925 (1961).

[33] T. Damour and K. Nordtvedt, Phys. Rev. D 48, 3436 (1993).

[34] J. Khoury and A. Weltman, Phys. Rev. D 69, 044026 (2004) astro-ph/0309411.

[35] S. Tsujikawa, K. Uddin, S. Mizuno, R. Tavakol and J. 'i. Yokoyama, Phys. Rev. D 77, 103009 (2008) arXiv:0803.1106 [astro-ph]].

[36] T. Chiba and M. Yamaguchi, JCAP 1310, 040 (2013) arXiv:1308.1142 [gr-qc]].

[37] C. Gordon, D. Wands, B. A. Bassett and R. Maartens, Phys. Rev. D 63, 023506 (2000) astro-ph/0009131.

[38] C. M. Peterson and M. Tegmark, Phys. Rev. D 87, 103507 (2013) arXiv:1111.0927 [astro-ph.CO]].

[39] V. Pettorino, Phys. Rev. D 88, 063519 (2013) arXiv:1305.7457 [astro-ph.CO]]. 